\title{
A Method for Electrical Property Tomography Based on a Three-Dimensional Integral Representation of the Electric Field
}

This paper was downloaded from TechRxiv (https://www.techrxiv.org).

\section{LICENSE}

CC BY 4.0

SUBMISSION DATE / POSTED DATE

$12-08-2021 / 13-08-2021$

\section{CITATION}

Eda, Naohiro; Fushimi,, Motofumi; Hasegawa, Keisuke; Nara, Takaaki (2021): A Method for Electrical Property Tomography Based on a Three-Dimensional Integral Representation of the Electric Field. TechRxiv. Preprint. https://doi.org/10.36227/techrxiv.15153579.v1

$\mathrm{DOI}$ 


\title{
A Method for Electrical Property Tomography Based on a Three-Dimensional Integral Representation of the Electric Field
}

\author{
Naohiro Eda, Motofumi Fushimi, Keisuke Hasegawa, Member, IEEE, and Takaaki Nara, Member, IEEE
}

\begin{abstract}
Magnetic resonance electrical properties tomography (MREPT) noninvasively reconstructs highresolution electrical property (EP) maps using MRI scanners and is useful for diagnosing cancerous tissues. However, conventional MREPT methods have limitations: sensitivity to noise in the numerical Laplacian operation, difficulty in reconstructing three-dimensional (3D) EPs and no guarantee of convergence in the iterative process. We propose a novel, iterative 3D reconstruction MREPT method without a numerical Laplacian operation. We derive an integral representation of the electric field using its Helmholtz decomposition with Maxwell's equations, under the assumption that the EPs are known on the boundary of the region of interest with the approximation that the unmeasurable magnetic field components are zero. Then, we solve the simultaneous equations composed of the integral representation and Ampere's law using a convex projection algorithm whose convergence is theoretically guaranteed. The efficacy of the proposed method was validated through numerical simulations and a phantom experiment. The results showed that this method is effective in reconstructing 3D EPs and is robust to noise. It was also shown that our proposed method with the unmeasurable component $\mathrm{H}^{-}$ enhances the accuracy of the EPs in a background and that with all the components of the magnetic field reduces the artifacts at the center of the slices except when all the components of the electric field are close to zero.
\end{abstract}

Index Terms-Magnetic resonance electrical properties tomography, magnetic resonance imaging, integral equation for the electric field, Helmholtz decomposition.

\section{INTRODUCTION}

$\mathbf{E}$ STIMATING the electrical properties (EPs) of tissues, such as permittivity and conductivity, is useful for the diagnosis of cancers, since cancerous tissue has abnormal EPs compared with normal tissues [1]. Additionally, the conductivity is essential for obtaining the specific absorption rate (SAR) for evaluating local hyperthermic tissue damage with radiofrequency energy [2]. For these reasons, many non-invasive methods have been developed to estimate EPs.

In electrical impedance tomography (EIT) [1] and magnetic induction tomography (MIT) [3], EP maps are calculated by

This work was supported by JSPS KAKENHI Grant Number $19 \mathrm{H} 04438$ and by Canon Medical Systems Corporation.

The authors are with the Graduate School of Information Science and Technology, University of Tokyo, Tokyo, Japan (email: naohiro-eda@g.ecc.u-tokyo.ac.jp; motofumi-

fushimi@g.ecc.u-tokyo.ac.jp; keisuke_hasegawa@ipc.i.utokyo.ac.jp; takaakinara@g.ecc.u-tokyo.ac.jp). injecting/inducing currents and by measuring the resultant electrical potential/magnetic field, respectively. However, the obtained EP maps have low spatial resolution due to the ill-posed nature of the inverse problem. Magnetic resonance electrical impedance tomography (MREIT) [4] addresses this issue, since the magnetic field inside the body can be measured using magnetic resonance image (MRI) scanners. However, it still requires the injection of a current into the human body, which degrades non-invasiveness since the signal-tonoise ratio (SNR) is determined by the amount of injected current. Furthermore, it is hard for MREIT to reconstruct the permittivities because of the use of low-frequency $(10 \mathrm{kHz})$ electromagnetic fields. On the other hand, in magnetic resonance electrical properties tomography (MREPT) [5], EP maps with high resolution can be reconstructed noninvasively from radio-frequency magnetic fields measured by MRI scanners.

The concept of MREPT was proposed by Haacke et al. [6] and Wen et al. [7]. Under the local homogeneity assumption that spatial variations of EPs are sufficiently small, EPs can be reconstructed from the B1 magnetic field $H^{+}:=\left(H_{x}+\right.$ $\left.i H_{y}\right) / 2$, where the $z$-axis is parallel to the body axis, measured by using B1-mapping techniques with a standard MRI scanner [8] [9] via the Helmholtz equation. This method is called standard-MREPT (std-MREPT). Katscher et al. [10] proposed a reconstruction method based on the integral expressions of Faraday's law and Ampere's law, assuming local homogeneity of EPs. However, it is shown that the assumption of local homogeneity of EPs leads to significant reconstruction errors in regions where EPs have spatial variations [11].

Various methods have been proposed to relax the local homogeneity assumption. Hafalir et al. [12] proposed convection-reaction MREPT (cr-MREPT), in which they derived the partial differential equation (PDE) for EPs and solved it by the finite element method (FEM) ignoring the $z$-axis variation of EPs. Although this method can obtain reconstruction results accounting for the spatial variations of EPs, it is sensitive to noise due to using the numerical Laplacian operation of the magnetic field, which amplifies noise.

Nara et al. [13] proposed an analytical EPT method without computing the Laplacian of $\mathrm{H}^{+}$: under a two-dimensional (2D) assumption that there is no $z$-axis variation of $\mathrm{H}^{+}$, an integral representation of the $z$-component of the electric field is derived by solving the so-called Dbar equation in complex analysis derived from Faraday's law. Combining this 
with Ampere's law, the EPs can be analytically reconstructed. Since this method using $E_{z}$ does not need to take derivation of Ampere's law as conventional methods do, it does not include a computation of the Laplacian of $H^{+}$. We call this method two-dimensional Dbar MREPT (2DD MREPT). Using the solution as an initial estimate, Nara et al. also proposed an iterative method (iterative 2DD MREPT) to account for the ignored term in 2DD. However, convergence is not guaranteed in this iterative process.

Balidemaj et al. and Leijsen et al. proposed contrast source inversion EPT (CSI-EPT) for 2D [14] and three-dimensional (3D) [15] cases, respectively, based on the CSI method which has been applied for the inverse scattering problem of the electromagnetic field. Hong et al. [16] and Guo et al. [17] also proposed 3D EPT methods with the same formulation based on different optimization methods. These methods calculate EPs iteratively such that they satisfy the integral equations for the measured magnetic field and the contrast source defined as the product of the electric field and the contrast function. However, the algorithm often converges to a local minimum without giving an appropriate initial estimate. Also, the high computation cost of iteratively solving the integral equations is a problem.

Liu et al. [18] proposed gradient-based EPT (gEPT), which accounts for the spatial variations of EPs with the transmit B1 field and receive B1 field measured by a multi-channel MRI. Wang et al. [19] then extended gEPT and proposed boundary informed EPT (BIEPT) given the EPs on the boundary of the ROI. These EPT methods with multi-channel MRI have the potential to obtain high-quality 3D reconstructions of EPs since these are free of the transceive phase approximation [10] and can reconstruct the receive field.

Data-based EPT methods (dictionary-based EPT [20] and DL-EPT [21]) have also been proposed, which obtain EPs from the relation between a database and measured data with local pattern matching and deep neural networks, respectively. However, the results strongly depend on the dictionaries or training dataset and do not often satisfy the physical constraint as they are not explicitly taken into consideration.

To summarize the problems of conventional EPT methods accounting for the variations of EPs with only the B1 magnetic field, the methods using the PDE for EPs require a numerical Laplacian operation, which leads to sensitivity to noise. Also, most of the conventional methods are limited to the 2D case. Even for the methods that apply for the 3D case, the computational cost is high and convergence is not guaranteed. In this paper, we develop a new iterative 3D EPT method. Our method estimates the electric field and EPs as in 2DD MREPT but in 3D space based on the Helmholtz decomposition of the electric field under the assumptions that the EPs are known on the boundary of the ROI, $H^{-}:=\left(H_{x}-i H_{y}\right) / 2$ and $H_{z}$ are negligible compared to $H^{+}$, and the transceive phase approximation [10] holds. Since our method does not take the derivative of Ampere's law, a computation of the Laplacian of the magnetic field is not essentially generated. Furthermore, the convergence of this algorithm is theoretically guaranteed. Although the assumption that EPs are known on the boundary of the ROI would be a limitation especially for in vivo experiments, a complementary use of std-MREPT to give the boundary EPs may overcome this defect. Also, a detailed comparison between the results of our proposed method with $\mathrm{H}^{+}$only, $\mathrm{H}^{+}$and $\mathrm{H}^{-}$, or all the components of the magnetic field conducted in this paper would clarify the importance of the measurement or computation of $H^{-}$and $H_{z}$ for MREPT.

The paper is organized as follows. In section IIA, a method for estimating the electric field from the magnetic field and EPs on the boundary of ROI is proposed. The approximation of the magnetic field in terms of the measured $H^{+}$is described in section IIB. A method for reconstructing EPs is then presented in section IIC. The settings for the numerical simulations and an experimental phantom are explained in section III, and the results of the proposed method are compared with those for the conventional methods in section IV. In section V, the features and problems of the proposed method are discussed. The paper is concluded in section VI.

\section{THEORY}

\section{A. Estimation of the 3D electric field based on the Helmholtz decomposition}

Let $\Omega \subset \mathbb{R}^{3}$ be a bounded domain representing an ROI in a human body and let $\partial \Omega$ be its boundary. Denote the admittivity to be reconstructed by $\gamma(\boldsymbol{r})=\sigma(\boldsymbol{r})+i \omega \epsilon(\boldsymbol{r})$, where $\sigma(\boldsymbol{r})$ and $\epsilon(\boldsymbol{r})$ are the conductivity and the permittivity, respectively, and $\omega$ is the Larmor frequency which is a fixed and known constant. The magnetic field $\boldsymbol{H}$ and the electric field $\boldsymbol{E}$ generated by a birdcage coil satisfy the time-harmonic Maxwell equations

$$
\begin{aligned}
\nabla \times \boldsymbol{E}(\boldsymbol{r}) & =-i \omega \mu \boldsymbol{H}(\boldsymbol{r}), \\
\nabla \times \boldsymbol{H}(\boldsymbol{r}) & =\gamma(\boldsymbol{r}) \boldsymbol{E}(\boldsymbol{r}),
\end{aligned}
$$

in $\Omega$, where $\mu$ is the permeability, which is assumed to be a constant and assumed to be equal to the free-space permeability. Eqs. (1) and (2) represent Faraday's law and Ampere's law, respectively. Although $\mathrm{H}^{+}$is the only measurable component in a typical MREPT setting, we assume that all components of $\boldsymbol{H}$ in $\Omega$ are known in this subsection, and how to approximate all the components of $\boldsymbol{H}$ is described in the next subsection. Throughout the paper, $\gamma$ on $\partial \Omega$ is assumed to be given as a boundary condition. Note that the external current applied to the birdcage coil is not included in the right-hand side of Eq. (2), since $\Omega$ is the ROI inside the body. In contrast to this, in CSI-EPT in [14]- [17], in order to exclude the external current from the governing equations, the so-called incident field that is generated by the external current in a background with known EPs should be considered. This is a major difference in the formulation of our method and those of [14]- [17].

Most of the conventional approaches such as [6], [7], [10], [12], [18], [19] and [22], solve the simultaneous equations composed of Eq. (1) and the derivative of Eq. (2). In fact, the relationship between the measured magnetic field and $\gamma$ is obtained by equating Eq. (1) and the rotation of Eq. (2), which necessitates computing the Laplacian of the magnetic field. In contrast, as shown in the following, our approach solves the simultaneous equations composed of Eq. (2) and 
an integral representation of $\boldsymbol{E}$ derived from Eqs. (1) and (2), which eliminates computation of the Laplacian of $\boldsymbol{H}$.

1) Integral representation of $\mathrm{E}$ : First, we derive an integral representation of $\boldsymbol{E}$. It is known that an arbitrary continuously differentiable vector field in a bounded domain $\Omega$ can be expressed by the Helmholtz decomposition [23] $\boldsymbol{f}=\boldsymbol{f}_{\text {irr }}+$ $f_{\text {sol }}$, where the irrotational part, $\boldsymbol{f}_{i r r}$, and the solenoidal part, $\boldsymbol{f}_{\text {sol }}$, are given by

$$
\begin{aligned}
\boldsymbol{f}_{i r r}=\nabla & \left(\int_{\partial \Omega}\left(\boldsymbol{n} \cdot \boldsymbol{f}\left(\boldsymbol{r}^{\prime}\right)\right) \frac{1}{4 \pi\left|\boldsymbol{r}-\boldsymbol{r}^{\prime}\right|} d S^{\prime}\right. \\
& \left.-\int_{\Omega}\left(\nabla^{\prime} \cdot \boldsymbol{f}\left(\boldsymbol{r}^{\prime}\right)\right) \frac{1}{4 \pi\left|\boldsymbol{r}-\boldsymbol{r}^{\prime}\right|} d V^{\prime}\right), \\
\boldsymbol{f}_{\text {sol }}=\nabla \times & \left(-\int_{\partial \Omega}\left(\boldsymbol{n} \times \boldsymbol{f}\left(\boldsymbol{r}^{\prime}\right)\right) \frac{1}{4 \pi\left|\boldsymbol{r}-\boldsymbol{r}^{\prime}\right|} d S^{\prime}\right. \\
& \left.+\int_{\Omega}\left(\nabla^{\prime} \times \boldsymbol{f}\left(\boldsymbol{r}^{\prime}\right)\right) \frac{1}{4 \pi\left|\boldsymbol{r}-\boldsymbol{r}^{\prime}\right|} d V^{\prime}\right),
\end{aligned}
$$

with $\boldsymbol{n}$ being an outward unit normal to $\partial \Omega$. Hence, setting $\boldsymbol{f}=\boldsymbol{E}$, we have

$$
\boldsymbol{E}=\boldsymbol{E}_{\text {irr }}+\boldsymbol{E}_{\text {sol }}
$$

with

$$
\begin{aligned}
\boldsymbol{E}_{i r r}(\boldsymbol{r})= & -\int_{\partial \Omega}\left(\boldsymbol{n} \cdot \boldsymbol{E}\left(\boldsymbol{r}^{\prime}\right)\right) \nabla^{\prime} \frac{1}{4 \pi\left|\boldsymbol{r}-\boldsymbol{r}^{\prime}\right|} d S^{\prime} \\
& +\int_{\Omega}\left(\nabla^{\prime} \cdot \boldsymbol{E}\left(\boldsymbol{r}^{\prime}\right)\right) \nabla^{\prime} \frac{1}{4 \pi\left|\boldsymbol{r}-\boldsymbol{r}^{\prime}\right|} d V^{\prime}, \\
\boldsymbol{E}_{\text {sol }}(\boldsymbol{r})= & -\int_{\partial \Omega}\left(\boldsymbol{n} \times \boldsymbol{E}\left(\boldsymbol{r}^{\prime}\right)\right) \times \nabla^{\prime} \frac{1}{4 \pi\left|\boldsymbol{r}-\boldsymbol{r}^{\prime}\right|} d S^{\prime} \\
& +\int_{\Omega}\left(\nabla^{\prime} \times \boldsymbol{E}\left(\boldsymbol{r}^{\prime}\right)\right) \times \nabla^{\prime} \frac{1}{4 \pi\left|\boldsymbol{r}-\boldsymbol{r}^{\prime}\right|} d V^{\prime} .
\end{aligned}
$$

To obtain Eqs. (6) and (7), we interchanged the order of the gradient or the curl operator and the integral in Eqs. (3) and (4). See Appendix A for derivation. Using Eqs. (1) and (2), Eq. (7) is written as

$$
\begin{aligned}
\boldsymbol{E}_{\text {sol }}(\boldsymbol{r})= & -\int_{\partial \Omega}\left(\boldsymbol{n} \times \frac{1}{\gamma}\left(\nabla^{\prime} \times \boldsymbol{H}\right)\right) \times \nabla^{\prime} \frac{1}{4 \pi\left|\boldsymbol{r}-\boldsymbol{r}^{\prime}\right|} d S^{\prime} \\
& -i \omega \mu \int_{\Omega} \boldsymbol{H} \times \nabla^{\prime} \frac{1}{4 \pi\left|\boldsymbol{r}-\boldsymbol{r}^{\prime}\right|} d V^{\prime},
\end{aligned}
$$

showing that the solenoidal part can be computed using the known values, $\boldsymbol{H}$ in $\Omega$ and $\gamma$ on $\partial \Omega$. On the other hand, the irrotational part (6) can be rewritten in terms of $\boldsymbol{E}$ in $\Omega$ only as

$$
\boldsymbol{E}_{i r r}(\boldsymbol{r})=-\int_{\Omega}\left(\boldsymbol{E}\left(\boldsymbol{r}^{\prime}\right) \cdot \nabla^{\prime}\right) \nabla^{\prime} \frac{1}{4 \pi\left|\boldsymbol{r}-\boldsymbol{r}^{\prime}\right|} d V^{\prime}
$$

See Appendix B for the detailed derivation. Hence, from Eqs. (5), (8) and (9), we have

$$
\boldsymbol{E}(\boldsymbol{r})=\boldsymbol{E}_{\text {sol }}(\boldsymbol{r})-\int_{\Omega}\left(\boldsymbol{E} \cdot \nabla^{\prime}\right) \nabla^{\prime} \frac{1}{4 \pi\left|\boldsymbol{r}-\boldsymbol{r}^{\prime}\right|} d V^{\prime} .
$$

This is an integral representation of $\boldsymbol{E}$ obtained from Eq. (1) with Eq. (2) for the boundary where $\boldsymbol{E}_{\text {sol }}$ is computed by the given values using Eq. (8).

Eq. (10) forms a contrast to our previous 2DD MREPT [13]: in the $2 \mathrm{D}$ case, the integral representation of the $z$ component of the electric field $E_{z}$ is obtained where $E_{z}$ is

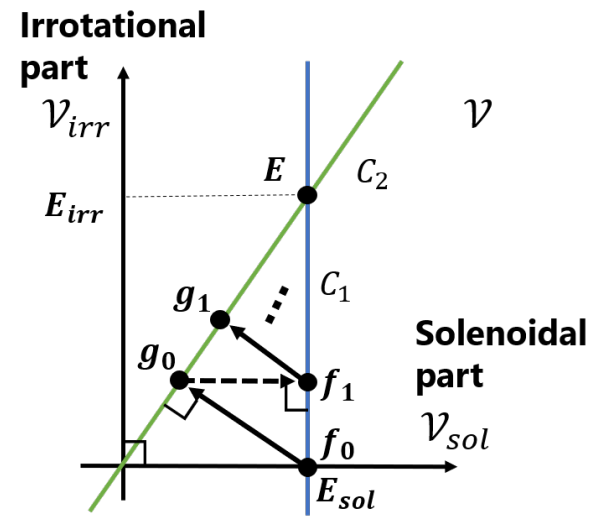

Fig. 1. Illustration of the problem and the proposed method. The electric field $\boldsymbol{E}$ is decomposed into a solenoidal part $\boldsymbol{E}_{\boldsymbol{s o l}}$ and irrotational part $\boldsymbol{E}_{\boldsymbol{i r r}}$. Our problem is to reconstruct $\boldsymbol{E}$ from $\boldsymbol{E}_{\boldsymbol{s o l}}$ computed in Eq.(8) from the known values. The blue and green lines are the vector spaces $\boldsymbol{C}_{1}$ and $\boldsymbol{C}_{2}$ in Eqs. (11) and (12), respectively. The solid and dashed arrows are the orthogonal projections onto $\boldsymbol{C}_{1}$ and $\boldsymbol{C}_{2}$ in Eqs. (13) and (15), respectively. The proposed method estimates $\boldsymbol{E} \in \boldsymbol{C}_{\mathbf{1}} \cap \boldsymbol{C}_{\mathbf{2}}$ by repeating these projections in turn. For definition of $\mathcal{V}, \mathcal{V}_{\text {sol }}$ and $\mathcal{V}_{\boldsymbol{i r r}}$, see Appendix D.

explicitly expressed in terms of $H^{+}$in $\Omega$ and $\gamma$ on $\partial \Omega$. Hence, substituting the obtained $E_{z}$ into Eq. (2) immediately determines $\gamma$. In contrast, Eq. (10) for the 3D case is not an explicit representation of $\boldsymbol{E}$ in terms of $\boldsymbol{H}$ in $\Omega$ and $\gamma$ on $\partial \Omega$, since the unknown $\boldsymbol{E}$ is still included in the right-hand side. Hence, we need to solve the simultaneous equations composed of the integral representation of $\boldsymbol{E}$ obtained in Eq. (10) and Ampere's law (2) for $\boldsymbol{E}$ and $\gamma$.

Remark 1. Eqs. (5), (6), and (7) are not applied to the case where $\gamma$ is piecewise constant, since in that case $\boldsymbol{E}$ is discontinuous at the boundary where EPs discontinuously change. Further analyses using the Helmholtz decomposition for piecewise continuously differentiable vector fields [24] are required for that case.

Remark 2. Although we consider $\boldsymbol{E}$ and $\boldsymbol{H}$ in Eqs. (1) and (2) only in $\Omega, \boldsymbol{E}_{i r r}$ in Eq. (6) and $\boldsymbol{E}_{\text {sol }}$ in Eq. (7) are defined in $\mathbb{R}^{3} / \Omega$ from $\boldsymbol{E}$ and $\boldsymbol{H}$ in $\Omega$. Here, it holds that $\boldsymbol{E}=\boldsymbol{E}_{\text {irr }}+$ $\boldsymbol{E}_{\text {sol }}=\mathbf{0}$ in $\mathbb{R}^{3} / \Omega$, which is shown in [25]. This means that, when using the Helmholtz decomposition of $\boldsymbol{E}$ in $\Omega, \boldsymbol{E}$ outside $\Omega$ is set to be zero, though the electric field physically exists outside $\Omega$. Hereafter, we regard $\boldsymbol{E}$ as the vector field that satisfies Eqs. (1) and (2) in $\Omega$ and is zero outside $\Omega$.

2) Solution to simultaneous equations composed of Eqs. (10) and (2): An iterative method to solve Eqs. (10) and (2) for $\boldsymbol{E}$ and $\gamma$ is proposed in the following. Let $\mathcal{W}$ be a vector space of vector fields which are supported on $\Omega$ and continuously differentiable in $\Omega$. Let $\mathcal{S}$ be a vector space of scalar fields whose support is $\Omega$. We define the set

$$
\begin{aligned}
C_{1}:= & \left\{\boldsymbol{f} \mid \boldsymbol{f}=\boldsymbol{E}_{\text {sol }}-\int_{\Omega}\left(\boldsymbol{g}\left(\boldsymbol{r}^{\prime}\right) \cdot \nabla^{\prime}\right) \nabla^{\prime} \frac{1}{4 \pi\left|\boldsymbol{r}-\boldsymbol{r}^{\prime}\right|} d V^{\prime},\right. \\
& \boldsymbol{g} \in \mathcal{W}\} .
\end{aligned}
$$

An element in $C_{1}$ is a vector field which is the summation of $\boldsymbol{E}_{\text {sol }}$ and an irrotational vector field in the form of Eq. (3), which is rewritten in the form of Eq. (9), for $\boldsymbol{g} \in \mathcal{W}$. Hence, $\boldsymbol{E}$ satisfying Eq. (10) is a member of $C_{1}$ for $\boldsymbol{g}=\boldsymbol{E}$. We 
define another set

$$
C_{2}:=\{\boldsymbol{f} \mid \boldsymbol{f}=a \nabla \times \boldsymbol{H}, a \in \mathcal{S}\} .
$$

An element in $C_{2}$ is a vector field which is expressed by $\nabla \times \boldsymbol{H}$ multiplied by an arbitrary scalar field $a$ supported on $\Omega$. Thus, $\boldsymbol{E}$ satisfying Eq. (2) is a member of $C_{2}$ when $a$ coincides with $1 / \gamma$ in $\Omega$ and is zero outside $\Omega$.

Our goal is to find an element of $C_{1} \cap C_{2}$ that satisfies both Eqs. (10) and (2). Given $\boldsymbol{E}_{\text {sol }}$, we need to recover $\boldsymbol{E}_{i r r}$ to obtain $\boldsymbol{E} \in C_{1} \cap C_{2}$. For this problem, we propose using a convex projection algorithm [26]. This method obtains a solution as an element in the intersection of sets representing the constraints that the solution should satisfy by alternating orthogonal projections onto each of them. When the sets are convex, the iteratively updated solution is guaranteed to converge to an element in the intersection of the convex sets. In our problem, starting from $\boldsymbol{E}_{\text {sol }}$ in Eq. (8), we repeat the orthogonal projections onto $C_{1}$ and $C_{2}$ in turn to renew the vector field, as schematically illustrated in Fig. 1. To be precise, we set the initial field as $\boldsymbol{f}_{0}:=\boldsymbol{E}_{\text {sol }}$. Then, for $\boldsymbol{f}_{n} \in C_{1}$ where $n=0,1, \ldots$, its projection onto $C_{2}$ is given by

$$
\boldsymbol{g}_{n}=a_{n} \nabla \times \boldsymbol{H}
$$

where

$$
a_{n}(\boldsymbol{r})=\left\{\begin{array}{ll}
\frac{\boldsymbol{f}_{n}(\boldsymbol{r}) \cdot(\nabla \times \boldsymbol{H}(\boldsymbol{r}))^{*}}{|\nabla \times \boldsymbol{H}(\boldsymbol{r})|^{2}} & (\boldsymbol{r} \in \Omega) \\
0 & (\boldsymbol{r} \notin \Omega)
\end{array} .\right.
$$

Then, for $\boldsymbol{g}_{n} \in C_{2}$ where $n=0,1, \ldots$, its projection onto $C_{1}$ is given by

$$
\boldsymbol{f}_{n+1}=\boldsymbol{E}_{\text {sol }}-\int_{\Omega}\left(\boldsymbol{g}_{n}\left(\boldsymbol{r}^{\prime}\right) \cdot \nabla^{\prime}\right) \nabla^{\prime} \frac{1}{4 \pi\left|\boldsymbol{r}-\boldsymbol{r}^{\prime}\right|} d V^{\prime} .
$$

We update the vector fields using the projections (13) and (15) iteratively.

Here, $C_{1}$ and $C_{2}$ are convex sets as proved in Appendix C. Also, as shown in Appendix D, it holds that

$$
<\boldsymbol{E}_{i r r}, \boldsymbol{E}_{\text {sol }}>_{\mathbb{R}^{3}} \equiv \int_{\mathbb{R}^{3}} \boldsymbol{E}_{i r r}(\boldsymbol{r}) \cdot \boldsymbol{E}_{\text {sol }}^{*}(\boldsymbol{r}) d V=0,
$$

where the dot product is defined as $\boldsymbol{f} \cdot \boldsymbol{g}=\sum_{i}\left(f_{i} g_{i}\right)(i=$ $x, y, z)$ with $\boldsymbol{f}$ and $\boldsymbol{g}$ being complex vector fields. Using Eq. (16), we can prove that the projections defined in Eqs. (13) and (15) are the orthogonal projections onto $C_{2}$ and $C_{1}$, respectively. See Appendix E for the proof. Hence, the conditions for convergence [26] are satisfied and $\boldsymbol{f}_{\infty} \equiv \lim _{n \rightarrow \infty} \boldsymbol{f}_{n}$ is guaranteed to be contained in $C_{1} \cap C_{2}$, which is a desired vector field.

In the implementation of this algorithm, it is not necessary to calculate the values in $\mathbb{R}^{3} \backslash \Omega$ since the projections (13) and (15) requires the vector fields in $\Omega$ only. Eq. (15) can be quickly calculated using an FFT since the volume integral in Eq. (15) expresses a convolution in $\Omega$ [27].

Remark 3. To show that the obtained $\boldsymbol{f}_{\infty}$ that satisfies both Eqs. (10) and (2) coincides with the true $\boldsymbol{E}$, we need to argue the uniqueness of the MREPT inverse problem: given $\boldsymbol{H}$ in $\Omega$, are $\gamma$ and $\boldsymbol{E}$ that satisfy Eqs. (1) and (2) uniquely determined?
Nachman's reconstruction formula [22] gives a partial answer: $\gamma$ is uniquely determined at locations where $\boldsymbol{H} \cdot(\nabla \times \boldsymbol{H}) \neq 0$. A further discussion for the locations where the condition is not met as well as an extension to the case where $\gamma$ on $\partial \Omega$ is given and/or only $H^{+}$is given instead of $\boldsymbol{H}$ are left for further studies.

\section{B. Approximation of all the components of the magnetic field}

In this subsection, we describe how to determine $\boldsymbol{H}$ and $\nabla \times \boldsymbol{H}$ to define the sets of vector fields (11) and (12) in the following three cases: (i) $\mathrm{H}^{+}, \mathrm{H}^{-}$and $H_{z}$ are given, (ii) $\mathrm{H}^{+}$ and $H^{-}$are given and (iii) $H^{+}$is given. In case (i), $\boldsymbol{H}$ and $\nabla \times \boldsymbol{H}$ can be rewritten as

$$
\begin{aligned}
\boldsymbol{H} & =\left[\begin{array}{c}
H^{+}+H^{-} \\
-i\left(H^{+}-H^{-}\right) \\
H_{z}
\end{array}\right], \\
\nabla \times \boldsymbol{H} & =\left[\begin{array}{c}
\partial_{y} H_{z}+i \partial_{z}\left(H^{+}-H^{-}\right) \\
\partial_{z}\left(H^{+}+H^{-}\right)-\partial_{x} H_{z} \\
-2 i \partial H^{+}+2 i \bar{\partial} H^{-}
\end{array}\right] \\
& =\left[\begin{array}{c}
\partial_{y} H_{z}+i \partial_{z}\left(H^{+}-H^{-}\right) \\
\partial_{z}\left(H^{+}+H^{-}\right)-\partial_{x} H_{z} \\
-4 i \partial H^{+}-i \partial_{z} H_{z}
\end{array}\right],
\end{aligned}
$$

where $\partial:=\left(\partial_{x}-i \partial_{y}\right) / 2, \bar{\partial}:=\left(\partial_{x}+i \partial_{y}\right) / 2$. See Appendix $\mathrm{F}$ for a detailed derivation.

In case (ii), we assume that $H_{z}$ generated by the end-ring is a minimum in the central regions for a birdcage coil [12]:

$$
H_{z}=0 \text {. }
$$

Under this assumption, $\boldsymbol{H}$ and $\nabla \times \boldsymbol{H}$ are approximately expressed with $\mathrm{H}^{+}$and $\mathrm{H}^{-}$as

$$
\boldsymbol{H} \simeq\left[\begin{array}{c}
H^{+}+H^{-} \\
-i\left(H^{+}-H^{-}\right) \\
0
\end{array}\right], \nabla \times \boldsymbol{H} \simeq\left[\begin{array}{c}
i \partial_{z}\left(H^{+}-H^{-}\right) \\
\partial_{z}\left(H^{+}+H^{-}\right) \\
-2 i \partial H^{+}+2 i \bar{\partial} H^{-}
\end{array}\right] .
$$

Finally, in case (iii), which is considered in most literatures, in addition to the assumption (19), we assume that $\left|H^{-}\right|$is much smaller than $\left|H^{+}\right|$due to quadrature excitation [10]:

$$
\left|H^{-}\right| \ll\left|H^{+}\right| .
$$

Under the assumptions (19) and (21), $\boldsymbol{H}$ and $\nabla \times \boldsymbol{H}$ are approximated with only $\mathrm{H}^{+}$to

$$
\boldsymbol{H} \simeq\left[\begin{array}{c}
H^{+} \\
-i H^{+} \\
0
\end{array}\right], \nabla \times \boldsymbol{H} \simeq\left[\begin{array}{c}
i \partial_{z} H^{+} \\
\partial_{z} H^{+} \\
-4 i \partial H^{+}
\end{array}\right] .
$$

In all cases, $\boldsymbol{E}$ is obtained via the convex projections onto the sets $C_{1}$ and $C_{2}$ in Eqs. (11) and (12) determined with $\boldsymbol{H}$ and $\nabla \times \boldsymbol{H}$. 


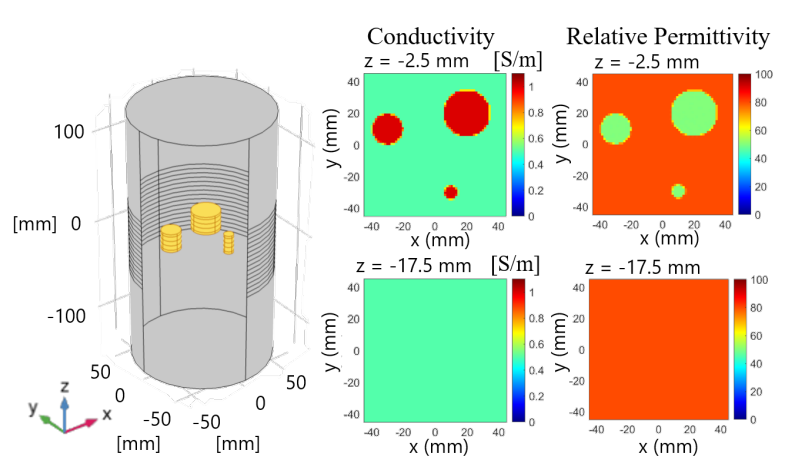

Fig. 2. Left: simulation phantom, consisting of three cylinders within a background cylinder. Right: EP maps of the model for the planes $z=$ $-\mathbf{2 . 5} \mathrm{mm}$ and $z=-\mathbf{1 7 . 5} \mathrm{mm}$

\section{Reconstruction of EPS}

In this subsection, we describe how to reconstruct the distributions of EPs by the estimated $\boldsymbol{E}$ and approximated $\boldsymbol{H}$. Using Ampere's law (Eq. (2)), we can obtain the value of EPs at each point in $\Omega$ from the ratio of the component of $\nabla \times \boldsymbol{H}$ and $\boldsymbol{E}$. Especially, when $E_{z} \neq 0$, using the $z$-component under the assumptions (19) and (21), we have

$$
\gamma=\frac{[\nabla \times \boldsymbol{H}]_{z}}{E_{z}} \simeq \frac{-4 i \partial H^{+}}{E_{z}}
$$

as in 2DD MREPT [13]. However, artifacts around the center of each $x y$-slice appear in 2DD MREPT since the $z$ component of the current, which corresponds to $E_{z}$, is small in this region as noted in the literature [12]. To resolve the problem, even when $E_{z}=0$ but $E_{x}$ and/or $E_{y}$ are not equal to zero, using all the components of Ampere's law, we can obtain $\gamma$ that minimizes $|\nabla \times \boldsymbol{H}-\gamma \boldsymbol{E}|^{2}$ as

$$
\gamma=\frac{|\nabla \times \boldsymbol{H}|^{2}}{\boldsymbol{E} \cdot(\nabla \times \boldsymbol{H})^{*}} .
$$

When the proposed method in section IIA converges to $\boldsymbol{f}_{\infty}=$ $\boldsymbol{E}$,

$$
a_{\infty}=\frac{\boldsymbol{f}_{\infty} \cdot(\nabla \times \boldsymbol{H})^{*}}{|\nabla \times \boldsymbol{H}|^{2}}=\frac{1}{\gamma} .
$$

Thus, $\gamma$ is automatically determined as $1 / a_{\infty}$ in the proposed method using all the components of the electric field.

\section{Simulation AND EXPERIMENT}

We verified our method using two numerical simulations and a phantom experiment. We compared our method with std-MREPT, 2DD MREPT, and iterative 2DD MREPT.

\section{A. Numerical simulations}

As shown in Fig. 2, we examined a simple model composed of three cylinders representing the domains with abnormal EPs (radii: $15 \mathrm{~mm}, 10 \mathrm{~mm}, 5 \mathrm{~mm}$, height: $20 \mathrm{~mm}(|z|<10$ $\mathrm{mm})$, centers: $(20 \mathrm{~mm}, 20 \mathrm{~mm}, 0 \mathrm{~mm}),(-30 \mathrm{~mm}, 10 \mathrm{~mm}$, $0 \mathrm{~mm}),(10 \mathrm{~mm},-30 \mathrm{~mm}, 0 \mathrm{~mm})$, conductivity: $1.0 \mathrm{~S} / \mathrm{m}$, relative permittivity: 50) in a large cylinder representing the background with normal EPs (radius: $72 \mathrm{~mm}$, height: $270 \mathrm{~mm}$

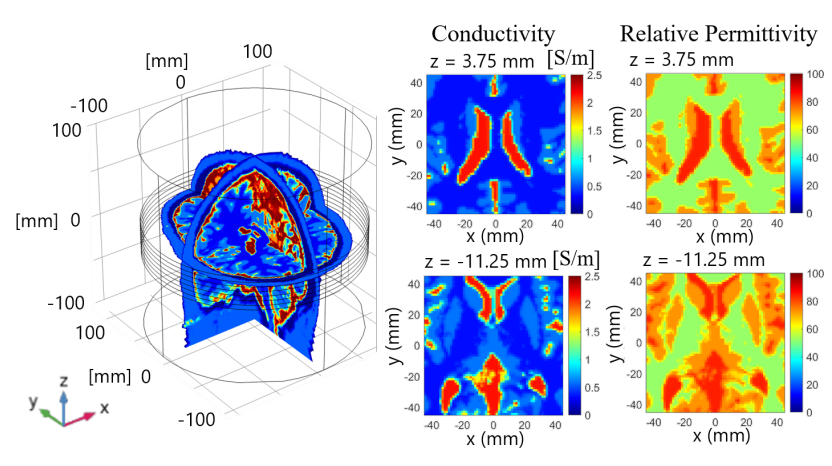

Fig. 3. Left: simulation phantom of the brain model. Right: EP maps of the model for the planes $z=\mathbf{3 . 7 5} \mathrm{mm}$ and $z=-\mathbf{1 1 . 2 5} \mathrm{mm}$.
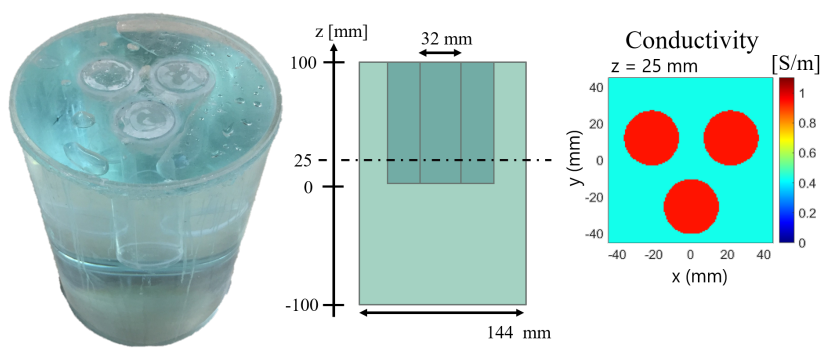

Fig. 4. Left: experimental phantom, consisting of three cylinders within a background cylinder. Middle: side view of the phantom. Right: conductivity map for the plane $z=\mathbf{2 5} \mathrm{mm}$ indicated by the dash-dotted line in the middle image.

$(|z|<135 \mathrm{~mm})$, center: origin, conductivity: $0.5 \mathrm{~S} / \mathrm{m}$, relative permittivity: 80). We call this model the 'cylindrical model'. As shown in Fig. 3, we also examined Aubert-Broche's model [28] representing a brain, which is acquired by high-resolution MRI imaging with a resolution of $1.4 \mathrm{~mm}$ and segmented into ten types of tissue. We simplified the model by reducing the number of tissue types to five: CSF, WM, GM, skull, and scalp as per the approach of Hafalir et al. [12]. The dimensions of the cuboid including the whole head are $177 \mathrm{~mm} \times 213 \mathrm{~mm}$ $\times 177 \mathrm{~mm}$. We call this model the 'brain model'.

The forward solutions were computed by FEM software (COMSOL Multiphysics, COMSOL Inc.) at 123.2 MHz using quadrature excitation. The resolutions of the cylindrical model in the $x$ and $y$-axis directions were about $1.4 \mathrm{~mm}$ and that in the $z$-axis direction was $5 \mathrm{~mm}$. The ROI of the cylindrical model was a $90 \mathrm{~mm} \times 90 \mathrm{~mm} \times 80 \mathrm{~mm}$ cuboid region (with a matrix size of $64 \times 64 \times 16$ ). The resolutions of the brain model in the $x$ and $y$-axis directions were about 1.4 $\mathrm{mm}$ and that in the $z$-axis direction was $2.5 \mathrm{~mm}$. The ROI of the brain model was a $90 \mathrm{~mm} \times 90 \mathrm{~mm} \times 40 \mathrm{~mm}$ cuboid region (with a matrix size of $64 \times 64 \times 16$ ). Gaussian noise was added to both the real and imaginary parts of the obtained $H^{+}, H^{-}$and $H_{z}$ data. The ratio of the standard deviations of the noise and that of the amplitude of each component was $1 \%$. To calculate the differential values of each component, we applied a $5 \times 5 \times 3$ Savitzky-Golay filter [29] to the data. All the reconstruction calculations were performed using MATLAB R2021a (The MathWorks Inc.) with an Intel(R) Core(TM) i7 $\mathrm{CPU}$ at $1.80 \mathrm{GHz}$ with $8 \mathrm{~GB}$ RAM. The process of estimating the electric field took about 3 minutes and the process of 
reconstructing EPs took less than 0.01 seconds. The true value of the EPs was assumed to be known on the boundary of the ROI. For the computation in iterative 2DD, five iterations were used. The iteration number of the proposed algorithm was fixed at 30 .

It is noted that the setting of the piecewise constant $\gamma$ seems to be contradictory to Remark 1 . However, when the magnetic field is sampled at discretized points, one cannot discriminate whether it is generated by a piecewise EP or a continuously differentiable EP. Hence, in the computations, we assume that the data originate from the continuously differentiable EP.

\section{B. Experimental phantom}

As shown in Fig. 4, we used an experimental phantom composed of three cylinders representing the domains with abnormal EPs (radius: $16 \mathrm{~mm}$, height: $100 \mathrm{~mm}(0<z<100$ $\mathrm{mm}$ ), conductivity: $0.94 \mathrm{~S} / \mathrm{m}$ ) in a large cylinder representing a background with normal EPs (radius: $72 \mathrm{~mm}$, height: $200 \mathrm{~mm}$ $(|z|<100 \mathrm{~mm})$, conductivity: $0.43 \mathrm{~S} / \mathrm{m})$. The inner and outer cylindrical containers were made of acrylic with a thickness of $3 \mathrm{~mm}$. The conductivity of each region was changed by adding $\mathrm{NaCl}$ to water. We measured the conductivity values using a conductivity meter (Hanna Instruments, HI 8733). The relative permittivity was homogeneous and equal to 80 which is that of water.

The data for the experimental phantom were measured using 3T MRI (SIEMENS, Magnetom Prisma) at 123.2 MHz using quadrature excitation. The amplitude of $\mathrm{H}^{+}$was calculated by the double angle method [8] from two gradient echo images with $60^{\circ}$ and $120^{\circ}$ flip angles. The phase of $\mathrm{H}^{+}$was calculated from two spin echo images with opposite readout directions under an approximation that the transmit and receive sensitivities are equal by using a switched birdcage coil [10]. The resolutions in the $x$ and $y$-axis directions were about 0.7 $\mathrm{mm}$ and that in the $z$-axis direction was $10 \mathrm{~mm}$. The ROI was a $90 \mathrm{~mm} \times 90 \mathrm{~mm} \times 80 \mathrm{~mm}$ cuboid region (with a matrix size of $128 \times 128 \times 8$ ). We estimated the SNR of the MRI signal as the ratio between the mean of the signal in the background region (with a matrix size of $30 \times 30 \times 8$ ) and the standard deviation of the signal in the air region (with a matrix size of $30 \times 30 \times 8$ ). Both the estimated SNRs of the gradient echo images and the spin echo images were about $0.5 \%$. We also used a Savitzky-Golay filter with a size $[9 \times 9 \times 3]$ for the phantom experiment using std-MREPT and $[5 \times 5 \times 3]$ for the other cases.

In both the numerical and phantom experiments, the results were evaluated using the total relative error of the reconstructed field defined using the $\mathrm{L}_{2}$-norm as

$$
\text { Error }_{x}:=\sqrt{\frac{\sum_{i}\left(x_{\mathrm{r}}\left(\boldsymbol{r}_{i}\right)-x_{\mathrm{t}}\left(\boldsymbol{r}_{i}\right)\right)^{2}}{\sum_{i}\left(x_{\mathrm{t}}\left(\boldsymbol{r}_{i}\right)\right)^{2}}},
$$

where $x_{\mathrm{r}}$ and $x_{\mathrm{t}}$ are the reconstructed and actual scalar field.

\section{Results}

\section{A. Numerical simulations}

Figs. 5 and 6 show the reconstruction results for the conductivity and the relative permittivity for the cylindrical model,

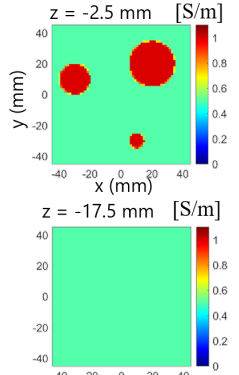

(a)

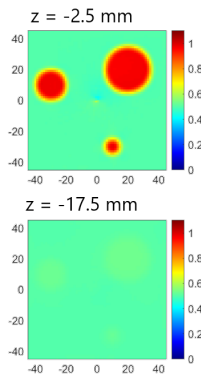

(e)

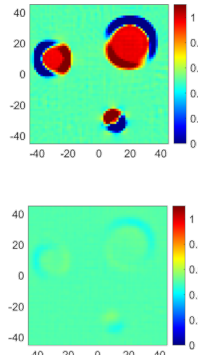

(b)
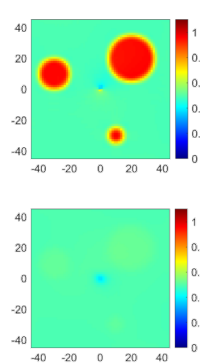

(f)
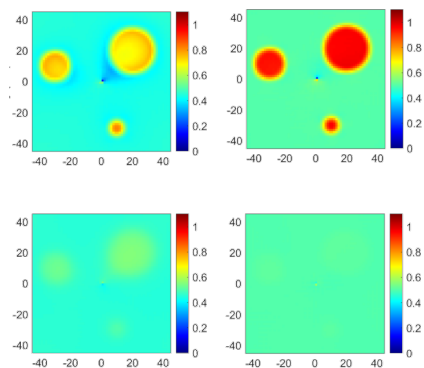

(c)

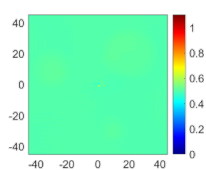

(d) g. 5econstruction results for the conductivity for the cylindrica model. The upper and lower rows show the results for slices at $z=$ $\mathbf{- 2 . 5} \mathrm{mm}$ and $\boldsymbol{z}=\mathbf{- 1 7 . 5} \mathrm{mm}$, respectively. (a) True conductivity, (b) reconstructed by std-MREPT, (c) reconstructed by 2DD, (d) reconstructed by iterative 2DD, (e)-(g) reconstructed by the proposed method with (e) all $\boldsymbol{H}$, (f) $\boldsymbol{H}^{+}$and $\boldsymbol{H}^{-}$and (g) $\boldsymbol{H}^{+}$only.

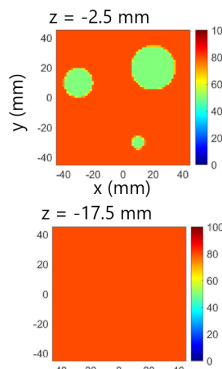

(a)

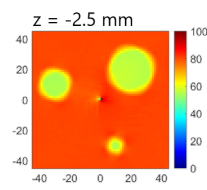

$z=-17.5 \mathrm{~mm}$

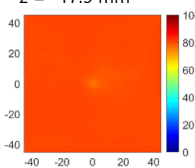

(e)
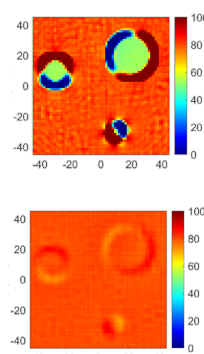

(b)
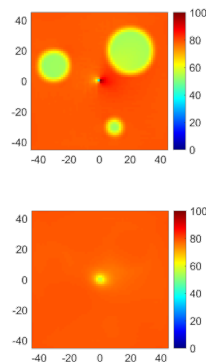

(f)
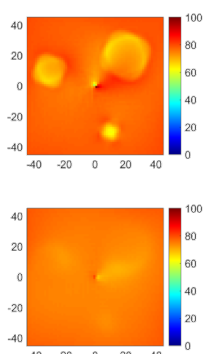

(c)
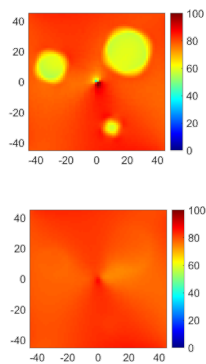

(g)

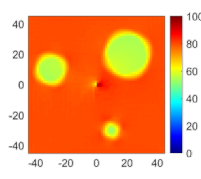

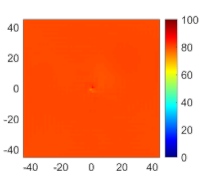

(d)
Fig. 6. Reconstruction results for the relative permittivity for the cylindrical model. The upper and lower rows show the results for slices at $\boldsymbol{z}=\mathbf{- 2 . 5} \mathrm{mm}$ and $\boldsymbol{z}=\mathbf{- 1 7 . 5} \mathrm{mm}$, respectively. (a) True relative permittivity, (b) reconstructed by std-MREPT, (c) reconstructed by 2DD, (d) reconstructed by iterative 2DD, (e)-(g) reconstructed by the proposed method with (e) all $\boldsymbol{H}$, (f) $\boldsymbol{H}^{+}$and $\boldsymbol{H}^{-}$and (g) $\boldsymbol{H}^{+}$only. 
TABLE I

TOTAL RELATIVE ERROR FOR THE CONDUCTIVITY AND THE RELATIVE PERMITTIVITY OF THE CYLINDRICAL MODEL

\begin{tabular}{c|ccccc}
\hline & \multirow{2}{*}{ 2DD } & iterative & \multicolumn{3}{c}{ the proposed method } \\
\cline { 4 - 6 } & & 2DD & all $\boldsymbol{H}$ & $H^{+}$and $H^{-}$ & $H^{+}$ \\
\hline \hline$\sigma$ (noise free) & $14.2 \%$ & $7.10 \%$ & $6.79 \%$ & $7.64 \%$ & $12.1 \%$ \\
$\epsilon_{r}$ (noise free) & $9.42 \%$ & $3.91 \%$ & $3.49 \%$ & $4.37 \%$ & $4.44 \%$ \\
\hline$\sigma$ (noise added) & $15.0 \%$ & $10.4 \%$ & $7.58 \%$ & $8.04 \%$ & $12.6 \%$ \\
$\epsilon_{r}$ (noise added) & $10.5 \%$ & $8.21 \%$ & $4.79 \%$ & $4.93 \%$ & $5.74 \%$ \\
\hline
\end{tabular}
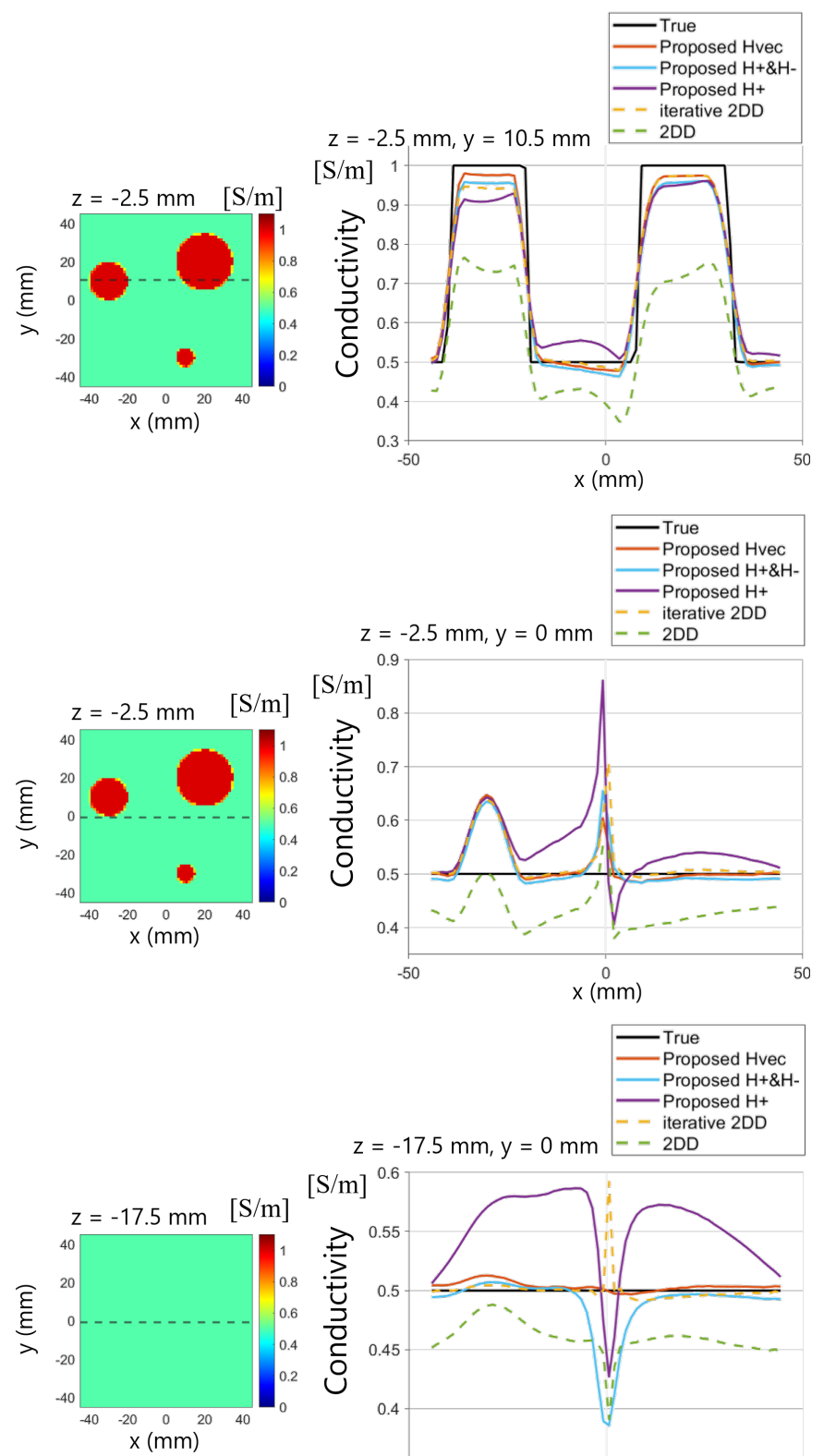

(a)
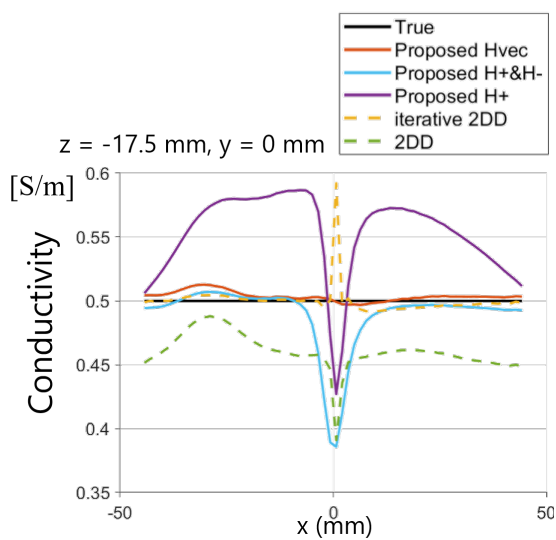

(b)

Fig. 7. Line profiles of the reconstructed conductivity for the cylindrical model. The upper and middle rows show the results at $\boldsymbol{y}=\mathbf{1 0 . 5} \mathrm{mm}$ and $y=0 \mathrm{~mm}$ along the $x$-axis for the slice at $z=-\mathbf{2 . 5} \mathrm{mm}$, and the lower row shows the result $\boldsymbol{y}=\mathbf{0} \mathrm{mm}$ along the $\boldsymbol{x}$-axis for the slice at $z=\mathbf{1 7 . 5} \mathrm{mm}$. (a) True conductivity map, (b) line profiles of the reconstructed and true conductivity.

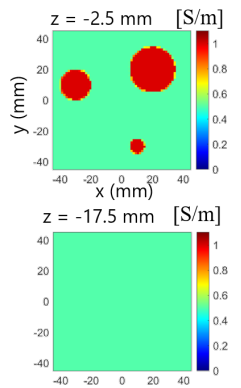

(a)

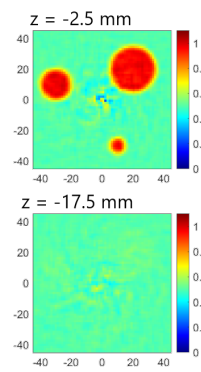

(e)
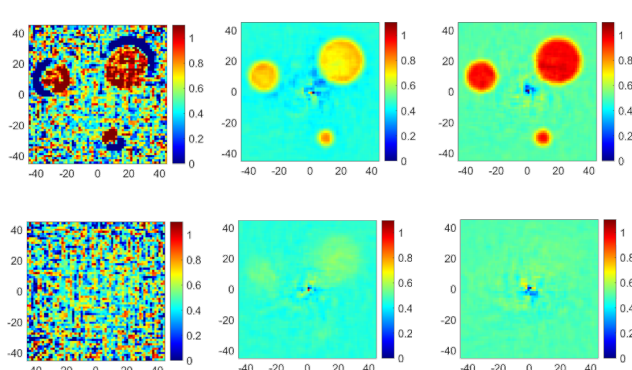

(b)

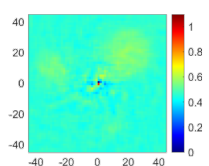

(c)

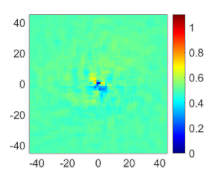

(d)
Fig. 8. Reconstruction results for the conductivity of the cylindrical model with $1 \%$ Gaussian noise. The upper and lower rows show the results for slices at $z=-\mathbf{2 . 5} \mathrm{mm}$ and $z=-\mathbf{1 7 . 5} \mathrm{mm}$, respectively. (a) True conductivity, (b) reconstructed by std-MREPT, (c) reconstructed by 2DD, (d) reconstructed by iterative 2DD, (e)-(g) reconstructed by the proposed method with (e) all $\boldsymbol{H}$, (f) $\boldsymbol{H}^{+}$and $\boldsymbol{H}^{-}$and (g) $\boldsymbol{H}^{+}$only.

respectively, without noise. Fig. 7 shows the line profiles of the conductivity. The proposed method in Fig. 5 (e)-(g) can stably reconstruct the conductivity on the boundary between the high-conductivity regions and the background since the local homogeneity of EPs is not assumed, in contrast to the assumption in std-MREPT. Compared with the 2DD method in Fig. 5 (c), the proposed method can give more accurate results by removing the assumption that $\partial_{z} H^{+}$is negligible, as also indicated by the line profiles in Fig. 7 and the total relative error for the conductivity in Table I. However, the iterative 2DD method in Fig. 5 (d) can give results with great accuracy. This shows that the iterative 2DD method works well when $\left|\partial_{z} H^{+}\right|$is not large, such as in these slices in the cylindrical model, as its effect is compensated by the iterations. As for the effect of the available components of $\boldsymbol{H}$, using $\mathrm{H}^{-}$ in addition to $H^{+}$improves reconstruction especially in the background, as shown by comparing Fig. 5 (f) and Fig. 5 (g), and is clearly seen in the line profiles at $z=-17.5 \mathrm{~mm}$ except around $x=0 \mathrm{~mm}$ in Fig. 7. Also, the proposed method with all $\boldsymbol{H}$ can suppress the artifacts around the center of the slice at $z=-17.5 \mathrm{~mm}$ and gives the best result in Fig. 5 (e) and Fig. 7. However, a spot-like artifact appears at the center of the slice at $z=-2.5 \mathrm{~mm}$ in Fig. 7 even if using all $\boldsymbol{H}$. The reason for this is discussed in section VB. The reconstruction results for the permittivity show nearly the same trend as those for the conductivity in Fig. 6. Fig. 8 shows the reconstruction results for the conductivity for the cylindrical model with $1 \%$ Gaussian noise added. The 2DD and the proposed method are robust to noise, as seen in Fig. 8 (c) and (e)-(g). However, in the iterative 2DD method as shown in Fig. 8 (d), the 
TABLE II

TOTAL RELATIVE ERROR FOR THE CONDUCTIVITY AND THE RELATIVE PERMITTIVITY OF THE BRAIN MODEL

\begin{tabular}{c|ccccc}
\hline & \multirow{2}{*}{$2 \mathrm{DD}$} & iterative & \multicolumn{3}{c}{ the proposed method } \\
\cline { 4 - 6 } & & 2DD & all $\boldsymbol{H}$ & $H^{+}$and $H^{-}$ & $H^{+}$ \\
\hline \hline$\sigma$ (noise free) & $57.8 \%$ & $89.2 \%$ & $27.9 \%$ & $32.2 \%$ & $35.2 \%$ \\
$\epsilon_{r}$ (noise free) & $46.1 \%$ & $97.4 \%$ & $11.2 \%$ & $15.4 \%$ & $20.9 \%$ \\
\hline$\sigma$ (noise added) & $57.9 \%$ & $90.8 \%$ & $28.0 \%$ & $32.3 \%$ & $35.3 \%$ \\
$\epsilon_{r}$ (noise added) & $46.1 \%$ & $96.7 \%$ & $11.5 \%$ & $15.6 \%$ & $21.2 \%$ \\
\hline
\end{tabular}

artifacts around the centers look like they were enhanced by the iterations compared with Fig. 5 (d). The reconstruction results for the relative permittivity have nearly the same trend as those for the conductivity.

Figs. 9 and 10 show the reconstruction results for the conductivity and the relative permittivity for the brain model. Fig. 11 shows the line profiles of the conductivity. As shown in Fig. 9 (c), the conductivity contrast reconstructed by the 2DD method is much lower than the true value. In the iterative 2DD method, the contrast becomes homogeneous due to the iterations, as seen in Fig. 9 (d). This is improved in the proposed method, as seen in Fig. 9 (e)-(g), due to no $2 \mathrm{D}$ assumption being applied, which is also indicated by the line profiles in Fig. 11. It is observed in Fig. 11 that the position and value of the peaks of the conductivity in std-MREPT do not often coincide with the true map. These results indicate the effectiveness of the proposed method for reconstructing the complicated 3D distributions of the EPs. However, we note that a high conductivity value for a small domain tends to be estimated to be lower by the proposed method. For example, the conductivity cannot be accurately estimated for a small domain with a width of about $5 \mathrm{~mm}$ at around $x=-40$ $\mathrm{mm}$ in Fig. 11 (b). This may be due to the smoothing effect of the Savitzky-Golay filter whose size for $[5 \times 5 \times 3]$ is 7.0 $\mathrm{mm} \times 7.0 \mathrm{~mm} \times 7.5 \mathrm{~mm}$. As for the effect of the available components of $\boldsymbol{H}$, the proposed method with $\mathrm{H}^{+}$and $\mathrm{H}^{-}$ can improve the results in the white matter (background). Furthermore, the proposed method with all $\boldsymbol{H}$ can suppress the artifacts around the center, especially in the result of the permittivity at $z=-11.25 \mathrm{~mm}$ in Fig. 10 (e) compared to Fig. 10 (f) and (g). We observe the same tendency for a comparison of the conductivity in Fig. 9 (e) and Fig. 9 (f) and $(\mathrm{g})$, and their line profiles in Fig. 11. Also, Fig. 12 shows the reconstruction results for the conductivity for the brain model with $1 \%$ Gaussian noise added, which have nearly the same trend as for the cylindrical model.

\section{B. Experimental phantom}

Fig. 13 shows the reconstruction results for the experimental phantom. Although the results for the 2DD method have lower value than the true values, as shown in Fig. 13 (c), the proposed method separates the background and the highconductivity regions more accurately as shown in Fig. 13 (e). In the iterative 2DD method, the artifacts at the centers of slices are spread by the iterations, especially at $z=-15$ $\mathrm{mm}$ in Fig. 13 (d) compared to those in Fig. 13 (c), which is the same tendency as for the cylindrical model with noise in Fig. 8. On the other hand, those artifacts at $z=-15 \mathrm{~mm}$

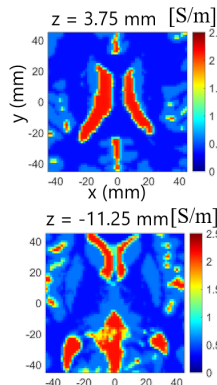

(a)

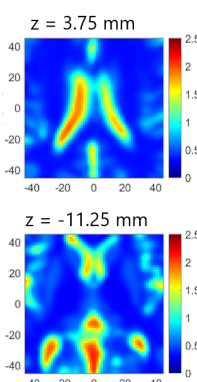

(e)

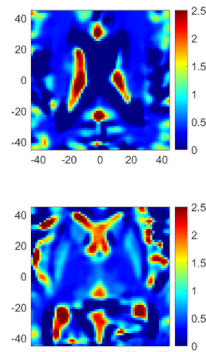

(b)
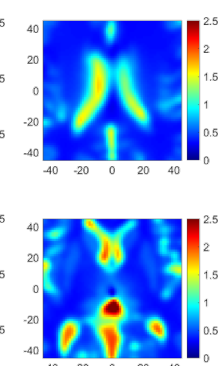

(f)
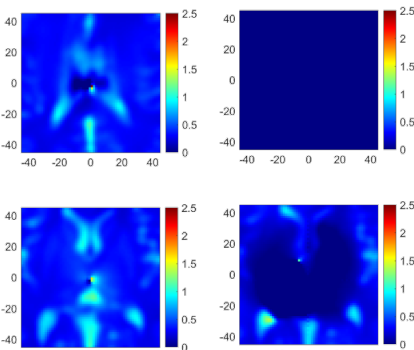

(c)

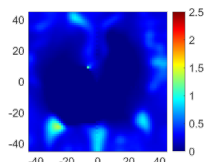

(d)
Fig. 9. Reconstruction results for the conductivity for the brain model. The upper and lower rows show the results for slices at $z=3.75 \mathrm{~mm}$ and $z=-\mathbf{1 1 . 2 5} \mathrm{mm}$, respectively. (a) True conductivity, (b) reconstructed by std-MREPT, (c) reconstructed by 2DD, (d) reconstructed by iterative 2DD, (e)-(g) reconstructed by the proposed method with (e) all $\boldsymbol{H}$, (f) $\boldsymbol{H}^{+}$and $\boldsymbol{H}^{-}$and (g) $\boldsymbol{H}^{+}$only.

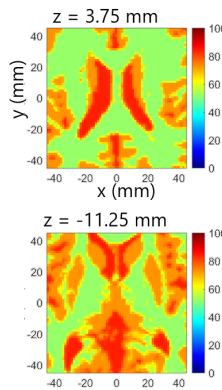

(a)

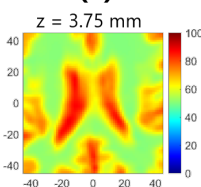

$\mathrm{z}=-11.25 \mathrm{~mm}$

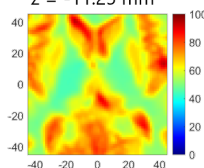

(e)
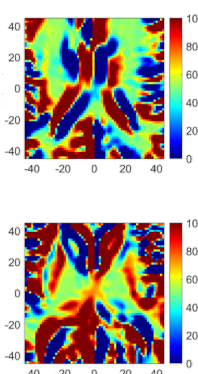

(b)
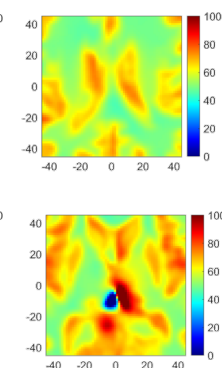

(f)
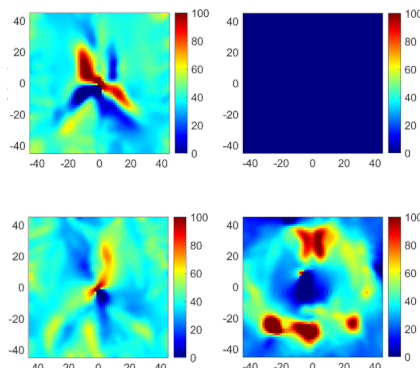

(c)

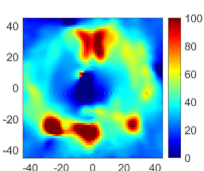

(d)
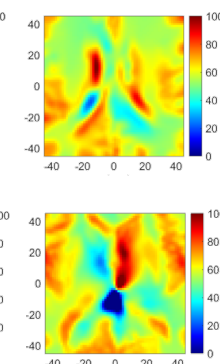

(g)

Fig. 10. Reconstruction results for the relative permittivity for the brain model. The upper and lower rows show the results for slices at $\boldsymbol{z}=\mathbf{3 . 7 5} \mathrm{mm}$ and $\boldsymbol{z}=\mathbf{- 1 1 . 2 5} \mathrm{mm}$, respectively. (a) True relative permittivity, (b) reconstructed by std-MREPT, (c) reconstructed by 2DD, (d) reconstructed by iterative $2 \mathrm{DD},(\mathrm{e})-(\mathrm{g})$ reconstructed by the proposed method with (e) all $\boldsymbol{H}$, (f) $\boldsymbol{H}^{+}$and $\boldsymbol{H}^{-}$and (g) $\boldsymbol{H}^{+}$only.

were less salient in the proposed method, as shown in Fig. 13 (e) as described for the simulations. This improvement is also confirmed at $z=25 \mathrm{~mm}$ : in a region where $|x|<5 \mathrm{~mm}$ and 


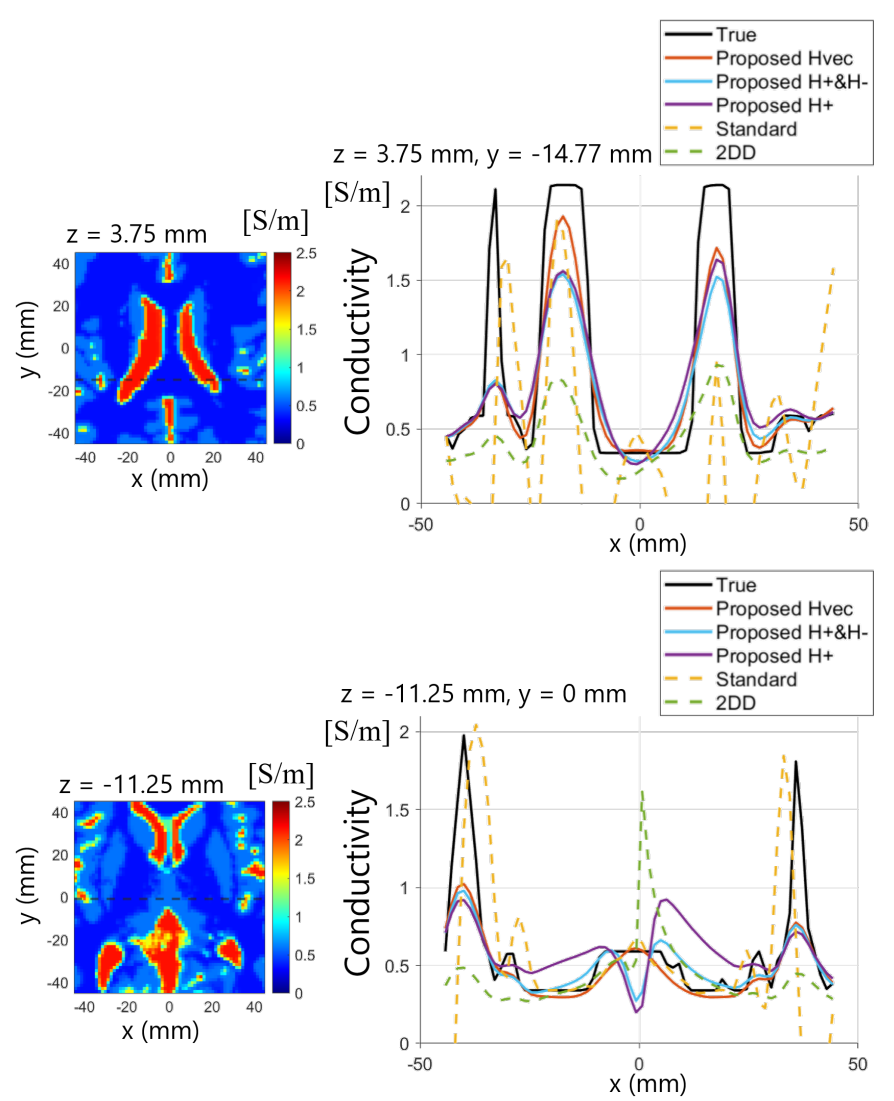

(a)

(b)

Fig. 11. Line profiles of the reconstructed conductivity for the brain model. The upper and lower rows show the results at $y=-\mathbf{1 4 . 7 7} \mathrm{mm}$ along the $\boldsymbol{x}$-axis for slices at $\boldsymbol{z}=\mathbf{3 . 7 5} \mathrm{mm}$ and at $\boldsymbol{y}=\mathbf{0} \mathrm{mm}$ along the $\boldsymbol{x}$-axis for slices at $\boldsymbol{z}=\mathbf{- 1 1 . 2 5} \mathrm{mm}$, respectively. (a) True conductivity map, (b) line profiles of the reconstructed and true conductivity.

$|y|<5 \mathrm{~mm}$, the reconstructed values are $\sigma \in[-0.93,12.14]$ $\mathrm{S} / \mathrm{m},[-7.72,3.07] \mathrm{S} / \mathrm{m}$, and $[-0.03,0.59] \mathrm{S} / \mathrm{m}$ for Fig. 13 (c), (d), and (e), respectively. Also, small high-frequency artifacts appear in the std-MREPT, as shown in Fig. 13 (b). This is due to enhancement of the noise in the data through a numerical computation of its Laplacian. It is noted that the proposed method gives large errors at the boundary between the background and the high-conductivity region at $z=25 \mathrm{~mm}$ as shown in Fig. 13 (e), although they are smaller compared than the result by the std-MREPT in Fig. 13 (b). This is because those regions in our phantom are separated with the acrylic cylinder with a thickness of $3 \mathrm{~mm}$ and hence the signal level there was very low.

\section{DISCUSSION}

\section{A. Improvement of accuracy for the electric field by adding information on $\mathrm{H}^{-}$and $\mathrm{H}_{z}$}

Fig. 14 shows the change in the relative errors for each component of $\boldsymbol{E}$ at $z=-2.5 \mathrm{~mm}$ and $z=-17.5 \mathrm{~mm}$ in the cylindrical model. Table III shows the total relative errors for each component of $\boldsymbol{E}$. Fig. 14 (b) and (e) show the results when $H^{-}$is given. We observe that the accuracy of the estimated $E_{z}$ is improved, while the accuracy of the

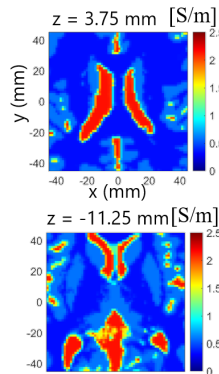

(a)

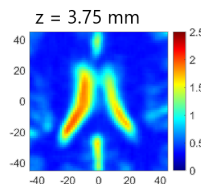$$
z=-11.25 \mathrm{~mm}
$$

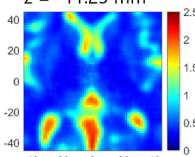

(e)

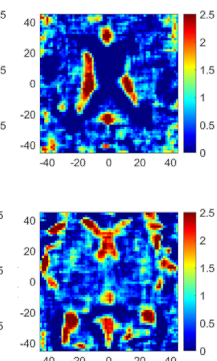

(b)
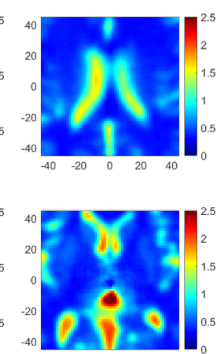

(f)
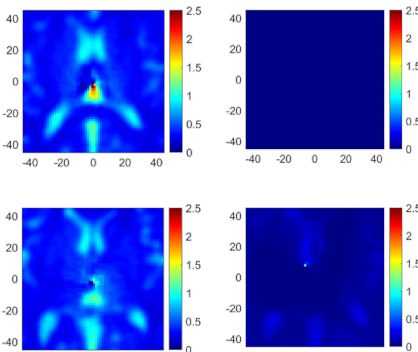

(c)

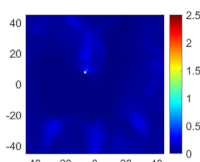

(d)
Fig. 12. Reconstruction results for the conductivity for the brain model with $1 \%$ Gaussian noise. The upper and lower rows show the results for slices at $z=\mathbf{3 . 7 5} \mathrm{mm}$ and $z=-\mathbf{1 1 . 2 5} \mathrm{mm}$, respectively. (a) True conductivity, (b) reconstructed by std-MREPT, (c) reconstructed by 2DD, (d) reconstructed by iterative $2 \mathrm{DD},(\mathrm{e})-(\mathrm{g})$ reconstructed by the proposed method with (e) all $\boldsymbol{H}$, (f) $\boldsymbol{H}^{+}$and $\boldsymbol{H}^{-}$and (g) $\boldsymbol{H}^{+}$only.

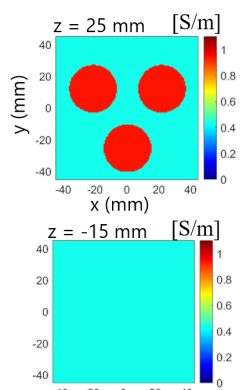

(a)
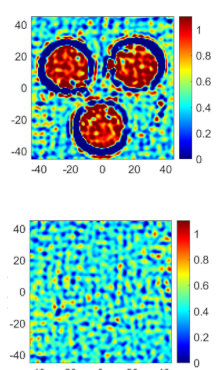

(b)
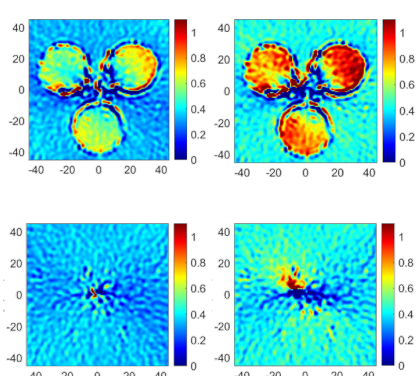

(c)

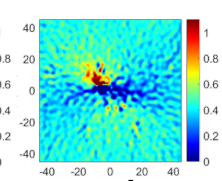

(d)

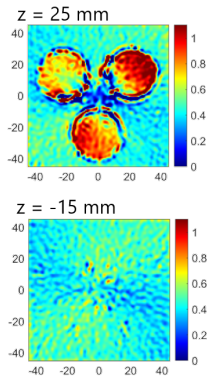

(e)

Fig. 13. Reconstruction results for the conductivity of the experimental phantom. The upper and lower rows show the results for slices at $z=25 \mathrm{~mm}$ and at $z=-\mathbf{1 5} \mathrm{mm}$, respectively. (a) True conductivity, (b) reconstructed by std-MREPT with $[9 \times 9 \times 3]$ Savitky-Golay filter, (c) reconstructed by $2 \mathrm{DD}$, (d) reconstructed by iterative 2DD, (e) reconstructed by the proposed method with $\boldsymbol{H}^{+}$only.

estimated $E_{x}, E_{y}$ cannot be improved, which is also indicated by the total relative errors in Table III. This improvement of the estimated $E_{z}$ may improve the reconstructed EPs for 


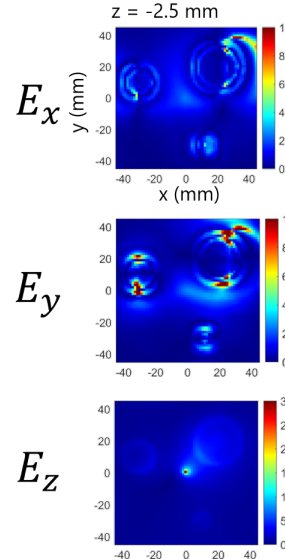

(a)
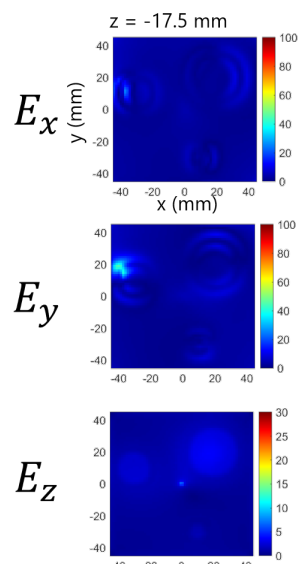

(d)
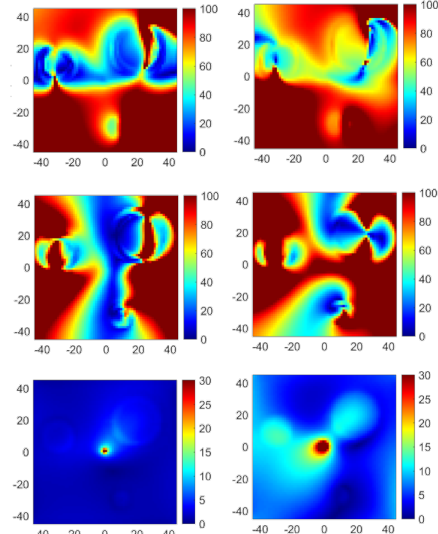

(b)
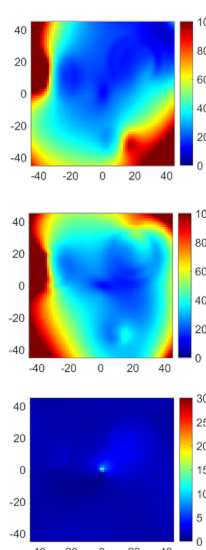

(e)

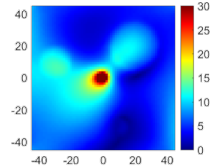

(c)
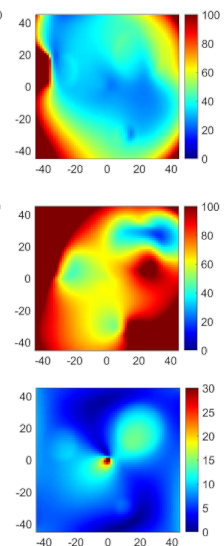

(f)
Fig. 14. Relative error for each component of the estimated $\boldsymbol{E}$ for the slice at $\boldsymbol{z}=\mathbf{- 2 . 5} \mathrm{mm}$ and $\boldsymbol{z}=\mathbf{- 1 7 . 5} \mathrm{mm}$ in the cylindrical model. (a)-(c) Results for the slice at $z=-\mathbf{2 . 5} \mathrm{mm}$, which includes the high-conductivity regions. (d)-(f) Results for the slice at $z=-\mathbf{1 7 . 5}$ $\mathrm{mm}$, where the conductivity is homogeneous. The upper row shows the results for $\boldsymbol{E}_{\boldsymbol{x}}$, the center row shows those for $\boldsymbol{E}_{\boldsymbol{y}}$ and the lower row shows those for $\boldsymbol{E}_{\boldsymbol{z}}$. (a),(d) Reconstructed with all $\boldsymbol{H}$, (b),(e) reconstructed with $\boldsymbol{H}^{+}$and $\boldsymbol{H}^{-}$, (c),(f) reconstructed with $\boldsymbol{H}^{+}$only.

the background in Fig. 5 (f) and Fig. 9 (f) mentioned in section IVA. Fig. 14 (a) and (d) show the results when all the components are given. It is found that the accuracy for each component of the estimated $\boldsymbol{E}$ can be improved for the entire ROI, which is also shown in Table III. It is considered that this accurate computation of all the components of $\boldsymbol{E}$ results in suppression of the artifacts around the centers in Fig. 5 (e) and Fig. 9 (e). Therefore, by adding information on all the components of $\boldsymbol{H}$, the accuracy of the estimated $E_{x}$, $E_{y}$ and $E_{z}$ can be improved, showing the potential for highly accurate reconstruction of EPs. For further improvements, the $H^{-}$reconstruction method proposed in [30] or the estimation of $H^{-}$with multi-channel MRI [18] will be combined with our proposed method in future studies.

\section{B. Suppression of the artifacts by the reconstruction with all the components of the electric field}

Fig. 15 shows the amplitude of each component of the actual electric field for the cylindrical model. It is observed
TABLE III

TOTAL RELATIVE ERROR FOR EACH COMPONENT OF $\boldsymbol{E}$ IN THE

\begin{tabular}{c|ccc}
\multicolumn{4}{|c}{ CYLINDRICAL MODEL } \\
\hline & all $\boldsymbol{H}$ & $H^{+}$and $H^{-}$ & $H^{+}$ \\
\hline \hline$E_{x}$ & $6.78 \%$ & $36.7 \%$ & $39.6 \%$ \\
\hline$E_{y}$ & $6.01 \%$ & $35.2 \%$ & $63.4 \%$ \\
\hline$E_{z}$ & $2.65 \%$ & $3.26 \%$ & $6.65 \%$ \\
\hline
\end{tabular}

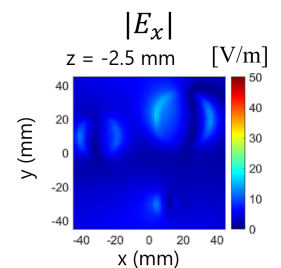

$\left|E_{y}\right|$

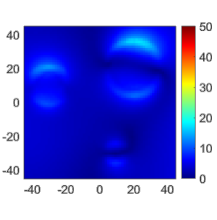

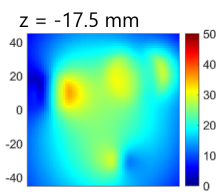

(a)

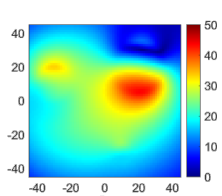

(b)
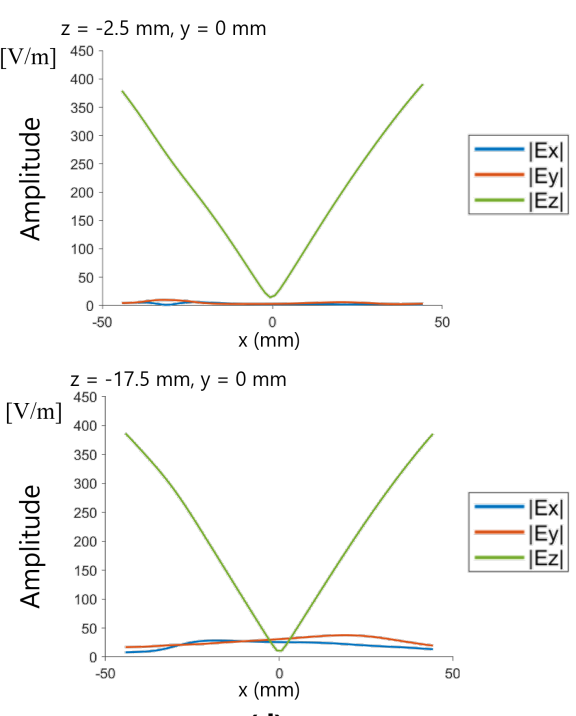

(d) (c)
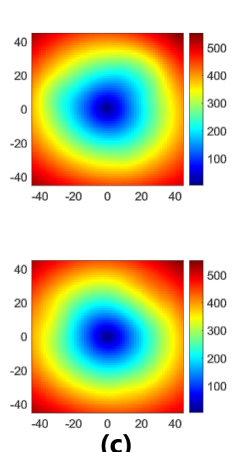

Fig. 15. Amplitude of each component of $\boldsymbol{E}$ for the cylindrical model. The upper and lower rows show the results for slices at $z=-\mathbf{2 . 5} \mathrm{mm}$ and at $\boldsymbol{z}=\mathbf{- 1 7 . 5} \mathrm{mm}$, respectively. (a) Amplitude of $\boldsymbol{E}_{\boldsymbol{x}}$, (b) amplitude of $\boldsymbol{E}_{\boldsymbol{y}}$, (c) amplitude of $\boldsymbol{E}_{\boldsymbol{z}}$, (d) line profiles along $\boldsymbol{x}$-axis at $\boldsymbol{y}=\mathbf{0}$.

that all the components of $\boldsymbol{E}$ are close to zero around the center of the slice at $z=-2.5 \mathrm{~mm}$. In this case, it is difficult to determine EP values even if using all components of $\boldsymbol{E}$. This is why the artifact is less suppressed around the center of the slice at $z=-2.5 \mathrm{~mm}$ in Figs. 5, 6, and 7. Also, Fig. 16 shows the amplitude of each component of the actual electric field for the brain model. Fig. 16 indicates that the amplitude of $E_{x}$ and $E_{y}$ is large around the centers of the slice at $z=3.75 \mathrm{~mm}$, although the amplitude of all the components of $\boldsymbol{E}$ is small around the centers of the slice at $z=-11.25 \mathrm{~mm}$. Correspondingly, the accuracy of the reconstructed EPs at the center of each slice in Fig. 9 and 10 is better improved at $z=3.75 \mathrm{~mm}$ than at $z=-11.25 \mathrm{~mm}$. Therefore, the reconstruction with all $\boldsymbol{E}$ is effective except 


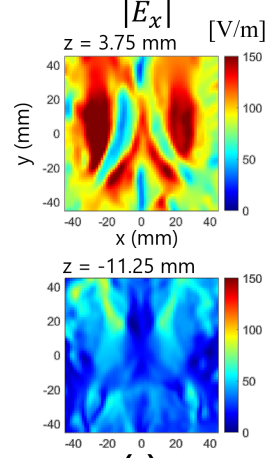

(a)
$\left|E_{y}\right|$
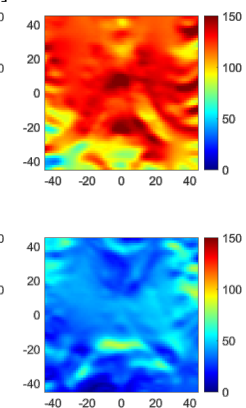

(b)

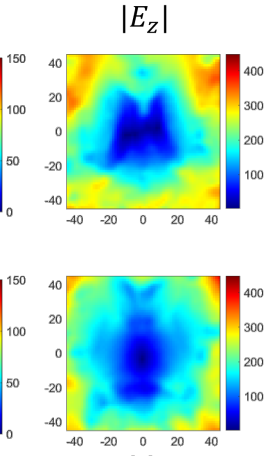

(c)
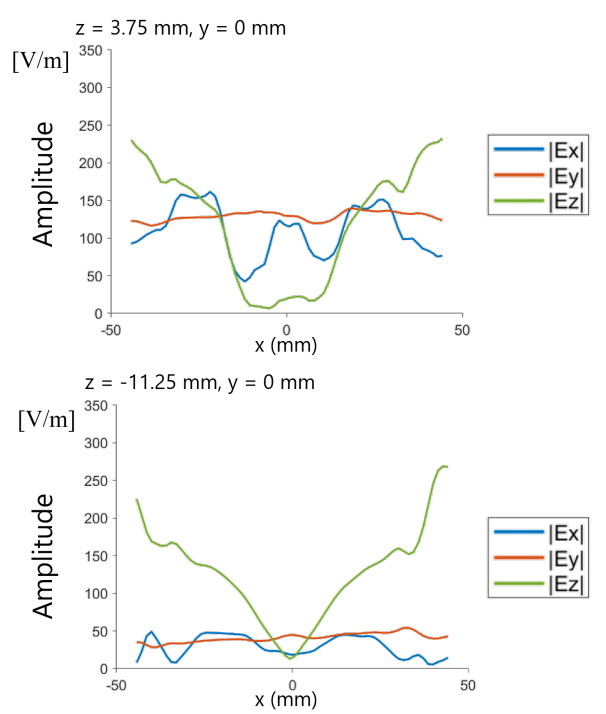

(d)

Fig. 16. Amplitude of each component of $\boldsymbol{E}$ for the brain model. The upper and lower rows show the results for slices at $z=3.75 \mathrm{~mm}$ and at $\boldsymbol{z}=\mathbf{- 1 1 . 2 5} \mathrm{mm}$, respectively. (a) Amplitude of $\boldsymbol{E}_{\boldsymbol{x}}$, (b) amplitude of $\boldsymbol{E}_{\boldsymbol{y}}$, (c) amplitude of $\boldsymbol{E}_{\boldsymbol{z}}$, (d) line profiles along $\boldsymbol{x}$-axis at $\boldsymbol{y}=\mathbf{0}$.

when the amplitude of all components of $\boldsymbol{E}$ is close to zero.

\section{CONCLUSION}

In this paper, we proposed a novel iterative 3D EPT method. Under the assumptions that the EP values are known on the boundary of the ROI, $H^{-}$and $H_{z}$ are negligible compared to $H^{+}$, and the transceive phase approximation holds, we proposed to solve the simultaneous equations composed of Ampere's law and an integral representation of the electric field derived from its Helmholtz decomposition and Maxwell's equations. Using a convex projection algorithm where the convergence is theoretically guaranteed, both the electric field and the EPs that satisfy the simultaneous equations are obtained. Our method was verified by numerical simulations and a phantom experiment and was found to be superior to the previous methods, the 2DD and iterative 2DD methods, in its effectiveness in reconstructing 3D EPs and robustness to noise. Our method achieved high-contrast reconstruction for complicated 3D EPs, such as those in a brain, and performed well with noisy simulation data and an experimental phantom. We compared three cases where all the components of the magnetic field are given, $\mathrm{H}^{+}$and $\mathrm{H}^{-}$are given, and $\mathrm{H}^{+}$only is given. It was observed that adding $H^{-}$reduced the artifacts in the background and using $H_{z}$ further improved the artifact at the centers of the slices except when all the components of the electric field are close to zero. The necessity of providing the EP values on the boundary of the ROI is a limitation of our method for in vivo applications. A possible approach is to obtain these values by std-MREPT. This will be an important aspect of future studies.

\section{APPENDIX}

\section{A. Derivation of Eqs.(6) and (7) from Eqs.(3) and (4)}

Noting that $\nabla^{\prime} \frac{1}{4 \pi\left|\boldsymbol{r}^{\prime}-\boldsymbol{r}\right|}=-\nabla \frac{1}{4 \pi\left|\boldsymbol{r}^{\prime}-\boldsymbol{r}\right|}, \boldsymbol{f}_{i r r}$ in Eq. (3) can be expressed as

$$
\begin{aligned}
\boldsymbol{f}_{i r r}(\boldsymbol{r})= & \int_{\partial \Omega} \nabla\left(\left(\boldsymbol{n} \cdot \boldsymbol{f}\left(\boldsymbol{r}^{\prime}\right)\right) \frac{1}{4 \pi\left|\boldsymbol{r}-\boldsymbol{r}^{\prime}\right|}\right) d S^{\prime} \\
& -\int_{\Omega} \nabla\left(\left(\nabla^{\prime} \cdot \boldsymbol{f}\left(\boldsymbol{r}^{\prime}\right)\right) \frac{1}{4 \pi\left|\boldsymbol{r}-\boldsymbol{r}^{\prime}\right|}\right) d V^{\prime} \\
= & \int_{\partial \Omega}(\boldsymbol{n} \cdot \boldsymbol{f}) \nabla \frac{1}{4 \pi\left|\boldsymbol{r}-\boldsymbol{r}^{\prime}\right|} d S^{\prime} \\
& -\int_{\Omega}\left(\nabla^{\prime} \cdot \boldsymbol{f}\right) \nabla \frac{1}{4 \pi\left|\boldsymbol{r}-\boldsymbol{r}^{\prime}\right|} d V^{\prime} \\
= & -\int_{\partial \Omega}(\boldsymbol{n} \cdot \boldsymbol{f}) \nabla^{\prime} \frac{1}{4 \pi\left|\boldsymbol{r}-\boldsymbol{r}^{\prime}\right|} d S^{\prime} \\
& +\int_{\Omega}\left(\nabla^{\prime} \cdot \boldsymbol{f}\right) \nabla^{\prime} \frac{1}{4 \pi\left|\boldsymbol{r}-\boldsymbol{r}^{\prime}\right|} d V^{\prime} .
\end{aligned}
$$

$f_{\text {sol }}$ in Eq. (4) can also be expressed as

$$
\begin{aligned}
\boldsymbol{f}_{\text {sol }}(\boldsymbol{r})= & -\int_{\partial \Omega} \nabla \times\left(\left(\boldsymbol{n} \times \boldsymbol{f}\left(\boldsymbol{r}^{\prime}\right)\right) \frac{1}{4 \pi\left|\boldsymbol{r}-\boldsymbol{r}^{\prime}\right|}\right) d S^{\prime} \\
& +\int_{\Omega} \nabla \times\left(\left(\nabla^{\prime} \times \boldsymbol{f}\left(\boldsymbol{r}^{\prime}\right)\right) \frac{1}{4 \pi\left|\boldsymbol{r}-\boldsymbol{r}^{\prime}\right|}\right) d V^{\prime} \\
= & -\int_{\partial \Omega} \nabla \frac{1}{4 \pi\left|\boldsymbol{r}-\boldsymbol{r}^{\prime}\right|} \times(\boldsymbol{n} \times \boldsymbol{f}) d S^{\prime} \\
& +\int_{\Omega} \nabla \frac{1}{4 \pi\left|\boldsymbol{r}-\boldsymbol{r}^{\prime}\right|} \times\left(\nabla^{\prime} \times \boldsymbol{f}\right) d V^{\prime} \\
= & -\int_{\partial \Omega}(\boldsymbol{n} \times \boldsymbol{f}) \times \nabla^{\prime} \frac{1}{4 \pi\left|\boldsymbol{r}-\boldsymbol{r}^{\prime}\right|} d S^{\prime} \\
& +\int_{\Omega}\left(\nabla^{\prime} \times \boldsymbol{f}\right) \times \nabla^{\prime} \frac{1}{4 \pi\left|\boldsymbol{r}-\boldsymbol{r}^{\prime}\right|} d V^{\prime} .
\end{aligned}
$$

Setting $\boldsymbol{f}=\boldsymbol{E}$ gives Eqs. (6) and (7).

\section{B. Derivation of Eq. (9)}

Using the vector calculus identity, it holds that

$$
\nabla \cdot\left(\boldsymbol{f} \partial_{i} G\right)=\nabla \cdot \boldsymbol{f} \partial_{i} G+\boldsymbol{f} \cdot \nabla \partial_{i} G,
$$

where $G$ is a scalar field and $i=x, y, z$. Integrated over $\Omega$ and using Gauss's theorem, Eq. (29) is transformed to

$$
\begin{aligned}
\int_{\Omega} \nabla \cdot\left(\boldsymbol{f} \partial_{i} G\right) d V & =\int_{\partial \Omega} \boldsymbol{f} \partial_{i} G \cdot \boldsymbol{n} d S \\
& =\int_{\Omega}\left(\nabla \cdot \boldsymbol{f} \partial_{i} G+\boldsymbol{f} \cdot \nabla \partial_{i} G\right) d V .
\end{aligned}
$$


Considering Eq. (30) for all $i$, we have

$$
\int_{\partial \Omega}(\boldsymbol{f} \cdot \boldsymbol{n}) \nabla G d S=\int_{\Omega}((\nabla \cdot \boldsymbol{f}) \nabla G+(\boldsymbol{f} \cdot \nabla) \nabla G) d V .
$$

Hence, $\boldsymbol{f}_{i r r}$ in Eq. (3) is rewritten as

$$
\boldsymbol{f}_{i r r}(\boldsymbol{r})=-\int_{\Omega}\left(\boldsymbol{f} \cdot \nabla^{\prime}\right) \nabla^{\prime} \frac{1}{4 \pi\left|\boldsymbol{r}-\boldsymbol{r}^{\prime}\right|} d V^{\prime} .
$$

Setting $\boldsymbol{f}=\boldsymbol{E}$ gives Eq. (9).

\section{Proof that the set $\mathrm{C}_{1}$ and $\mathrm{C}_{2}$ are convex}

Let $f$ be a vector field expressed as

$$
\boldsymbol{f}=(1-\lambda) \boldsymbol{f}_{1}+\lambda \boldsymbol{f}_{2},
$$

where $\boldsymbol{f}_{1}, \boldsymbol{f}_{2} \in C_{1}$ and $\lambda \in[0,1]$. Since $\boldsymbol{f}_{1}, \boldsymbol{f}_{2} \in C_{1}$, these vector fields can be rewritten as

$$
\begin{aligned}
& \boldsymbol{f}_{1}=\boldsymbol{E}_{\text {sol }}-\int_{\Omega}\left(\boldsymbol{g}_{1} \cdot \nabla^{\prime}\right) \nabla^{\prime} \frac{1}{4 \pi\left|\boldsymbol{r}-\boldsymbol{r}^{\prime}\right|} d V^{\prime}, \\
& \boldsymbol{f}_{2}=\boldsymbol{E}_{\text {sol }}-\int_{\Omega}\left(\boldsymbol{g}_{2} \cdot \nabla^{\prime}\right) \nabla^{\prime} \frac{1}{4 \pi\left|\boldsymbol{r}-\boldsymbol{r}^{\prime}\right|} d V^{\prime} .
\end{aligned}
$$

Therefore, $f$ can be rewritten as

$$
\begin{aligned}
\boldsymbol{f}= & (1-\lambda)\left(\boldsymbol{E}_{\text {sol }}-\int_{\Omega}\left(\boldsymbol{g}_{1} \cdot \nabla^{\prime}\right) \nabla^{\prime} \frac{1}{4 \pi\left|\boldsymbol{r}-\boldsymbol{r}^{\prime}\right|} d V^{\prime}\right) \\
& +\lambda\left(\boldsymbol{E}_{\text {sol }}-\int_{\Omega}\left(\boldsymbol{g}_{2} \cdot \nabla^{\prime}\right) \nabla^{\prime} \frac{1}{4 \pi\left|\boldsymbol{r}-\boldsymbol{r}^{\prime}\right|} d V^{\prime}\right) \\
= & \boldsymbol{E}_{\text {sol }}-\int_{\Omega}\left(\left((1-\lambda) \boldsymbol{g}_{1}+\lambda \boldsymbol{g}_{2}\right) \cdot \nabla^{\prime}\right) \nabla^{\prime} \frac{1}{4 \pi\left|\boldsymbol{r}-\boldsymbol{r}^{\prime}\right|} d V^{\prime},
\end{aligned}
$$

which is included in $C_{1}$. Hence, $C_{1}$ is a convex set.

In a similar way, let $\boldsymbol{g}$ be a vector field expressed as

$$
\boldsymbol{g}=(1-\lambda) \boldsymbol{g}_{1}+\lambda \boldsymbol{g}_{2},
$$

where $\boldsymbol{g}_{1}, \boldsymbol{g}_{2} \in C_{2}$ and $\lambda \in[0,1]$. Since $\boldsymbol{g}_{1}, \boldsymbol{g}_{2} \in C_{2}$, these vector fields can be rewritten as

$$
\begin{aligned}
& \boldsymbol{g}_{1}=a_{1} \nabla \times \boldsymbol{H}, \\
& \boldsymbol{g}_{2}=a_{2} \nabla \times \boldsymbol{H},
\end{aligned}
$$

where $a_{1}, a_{2}$ are scalar fields supported on $\Omega$. Therefore, $\boldsymbol{g}$ can be rewritten as

$$
\boldsymbol{g}=\left((1-\lambda) a_{1}+\lambda a_{2}\right) \nabla \times \boldsymbol{H} .
$$

Because the support of $(1-\lambda) a_{1}+\lambda a_{2}$ is $\Omega, \boldsymbol{g}$ is included in $C_{2}$. Hence, $C_{2}$ is a convex set.

\section{Orthogonality in Eq. (16)}

Let $\mathcal{V}$ be a vector space of vector fields which are continuously differentiable in $\Omega$ and $\mathbb{R}^{3} / \Omega$ and are vanishing at enough quick rate for $|\boldsymbol{r}| \rightarrow \infty$. Note that $\boldsymbol{f} \in \mathcal{V}$ can be discontinuous on $\partial \Omega$. The Helmholtz decomposition of $\boldsymbol{f} \in \mathcal{V}$ is given [24] by $\boldsymbol{f}=\boldsymbol{f}_{\text {irr }, \mathbb{R}^{3}}+\boldsymbol{f}_{\text {sol }, \mathbb{R}^{3}}$ with

$$
\begin{aligned}
\boldsymbol{f}_{i r r, \mathbb{R}^{3}}(\boldsymbol{r})=\nabla & \left(\int_{\partial \Omega} \frac{\boldsymbol{n} \cdot\left(\boldsymbol{f}_{\Omega}\left(\boldsymbol{r}^{\prime}\right)-\boldsymbol{f}_{\mathbb{R}^{3} / \Omega}\left(\boldsymbol{r}^{\prime}\right)\right)}{4 \pi\left|\boldsymbol{r}-\boldsymbol{r}^{\prime}\right|} d S^{\prime}\right. \\
& \left.-\int_{\mathbb{R}^{3}} \frac{\nabla^{\prime} \cdot \boldsymbol{f}\left(\boldsymbol{r}^{\prime}\right)}{4 \pi\left|\boldsymbol{r}-\boldsymbol{r}^{\prime}\right|} d V^{\prime}\right), \\
\boldsymbol{f}_{\text {sol, } \mathbb{R}^{3}}(\boldsymbol{r})=\nabla \times & \left(-\int_{\partial \Omega} \frac{\boldsymbol{n} \times\left(\boldsymbol{f}_{\Omega}\left(\boldsymbol{r}^{\prime}\right)-\boldsymbol{f}_{\mathbb{R}^{3} / \Omega}\left(\boldsymbol{r}^{\prime}\right)\right)}{4 \pi\left|\boldsymbol{r}-\boldsymbol{r}^{\prime}\right|} d S^{\prime}\right. \\
& \left.+\int_{\mathbb{R}^{3}} \frac{\nabla^{\prime} \times \boldsymbol{f}\left(\boldsymbol{r}^{\prime}\right)}{4 \pi\left|\boldsymbol{r}-\boldsymbol{r}^{\prime}\right|} d V^{\prime}\right),
\end{aligned}
$$

where $\boldsymbol{n}$ is an outward unit normal to $\partial \Omega$ and $\boldsymbol{f}_{\Omega}-\boldsymbol{f}_{\mathbb{R}^{3} / \Omega}$ represents the jump of $\boldsymbol{f}$ across $\partial \Omega$. We call $\boldsymbol{f}_{\text {irr }, \mathbb{R}^{3}}$ in Eq. (41) and $f_{\text {sol, } \mathbb{R}^{3}}$ in Eq. (42) the irrotational part and the solenoidal part of $\boldsymbol{f} \in \mathcal{V}$, and denote the sets of those vector fields for an arbitrary $f \in \mathcal{V}$ by $\mathcal{V}_{i r r}$ and $\mathcal{V}_{\text {sol }}$, respectively. Following the argument in [24], for $\boldsymbol{f}, \boldsymbol{g} \in \mathcal{V}$, it holds from the condition on $\boldsymbol{f}$ and $\boldsymbol{g}$ for $|\boldsymbol{r}| \rightarrow \infty$ that

$$
<\boldsymbol{f}_{i r r, \mathbb{R}^{3}}, \boldsymbol{g}_{\text {sol }, \mathbb{R}^{3}}>_{\mathbb{R}^{3}} \equiv \int_{\mathbb{R}^{3}} \boldsymbol{f}_{i r r, \mathbb{R}^{3}} \cdot \boldsymbol{g}_{\text {sol }, \mathbb{R}^{3}}^{*} d V=0,
$$

where $\boldsymbol{f}_{i r r, \mathbb{R}^{3}} \in \mathcal{V}_{i r r}$ is the irrotational part of $\boldsymbol{f}$ and $\boldsymbol{g}_{s o l, \mathbb{R}^{3}} \in$ $\mathcal{V}_{\text {sol }}$ is the solenoidal part of $\boldsymbol{g}$.

When the support of $\boldsymbol{f} \in \mathcal{V}$ is $\Omega, \boldsymbol{f}_{\mathbb{R}^{3} / \Omega}=\mathbf{0}$, and hence Eq. (41) turns to

$$
\begin{aligned}
\boldsymbol{f}_{i r r, \mathbb{R}^{3}}(\boldsymbol{r})=\nabla & \left(\int_{\partial \Omega}\left(\boldsymbol{n} \cdot \boldsymbol{f}_{\Omega}\left(\boldsymbol{r}^{\prime}\right)\right) \frac{1}{4 \pi\left|\boldsymbol{r}-\boldsymbol{r}^{\prime}\right|} d S^{\prime}\right. \\
& \left.-\int_{\Omega}\left(\nabla^{\prime} \cdot \boldsymbol{f}\left(\boldsymbol{r}^{\prime}\right)\right) \frac{1}{4 \pi\left|\boldsymbol{r}-\boldsymbol{r}^{\prime}\right|} d V^{\prime}\right),
\end{aligned}
$$

which is equal to $\boldsymbol{f}_{\text {irr }}$ in Eq. (3). Similarly, $\boldsymbol{f}_{\text {sol, } \mathbb{R}^{3}}=\boldsymbol{f}_{\text {sol }}$ in Eq. (4). In other words, Eqs. (3) and (4) can be regarded as the irrotational and the solenoidal part of $f \in \mathcal{V}$ supported on $\Omega$, respectively. Hence, for $\boldsymbol{f}_{i r r}$ and $\boldsymbol{f}_{\text {sol }}$ in Eqs. (3) and (4), it holds from Eq. (43) that

$$
<\boldsymbol{f}_{\text {irr }}, \boldsymbol{f}_{\text {sol }}>_{\mathbb{R}^{3}}=0 .
$$

Eq. (16) follows by setting $\boldsymbol{f}=\boldsymbol{E}$ whose support is $\Omega$ as mentioned in Remark 2.

\section{E. Proof that the projections defined in Eqs. (13) and (15) are the orthogonal projections}

Let us consider first the projection from $C_{1}$ to $C_{2}$ defined in Eq. (13). For an arbitrary vector field $\boldsymbol{g}^{\prime}=a^{\prime} \nabla \times \boldsymbol{H} \in C_{2}$, noting that $a_{n}$ in Eq. (14) minimizes $\left|\boldsymbol{f}_{n}-a \nabla \times \boldsymbol{H}\right|^{2}$, we have

$$
\begin{aligned}
\left\|\boldsymbol{f}_{n}-\boldsymbol{g}^{\prime}\right\|^{2} & =\int_{\mathbb{R}^{3}}\left|\boldsymbol{f}_{n}-a^{\prime} \nabla \times \boldsymbol{H}\right|^{2} d V+\int_{\mathbb{R}^{3} \backslash \Omega}\left|\boldsymbol{f}_{n}\right|^{2} d V \\
& \geq \int_{\mathbb{R}^{3}}\left|\boldsymbol{f}_{n}-a_{n} \nabla \times \boldsymbol{H}\right|^{2} d V+\int_{\mathbb{R}^{3} \backslash \Omega}\left|\boldsymbol{f}_{n}\right|^{2} d V \\
& =\left\|\boldsymbol{f}_{n}-\boldsymbol{g}_{n}\right\|^{2} .
\end{aligned}
$$

Thus, $\boldsymbol{g}_{n}$ is the nearest neighbor of $\boldsymbol{f}_{n}$ in $C_{2}$, showing that the projection defined in Eq. (13) is the orthogonal projection onto $C_{2}$.

Next, we consider the projection from $C_{2}$ to $C_{1}$ defined in Eq. (15). For an arbitrary vector field $\boldsymbol{f}^{\prime}=\boldsymbol{E}_{s o l}+\boldsymbol{f}_{i r r}^{\prime} \in C_{1}$, 
where $\boldsymbol{f}_{i r r}^{\prime}$ is the irrotational part of $\boldsymbol{f}^{\prime}$, it holds from Eq. (45) that

$$
\begin{aligned}
\left\|\boldsymbol{f}^{\prime}-\boldsymbol{g}_{n}\right\|^{2} & =\left\|\boldsymbol{f}_{i r r}^{\prime}-\boldsymbol{g}_{n, i r r}\right\|^{2}+\left\|\boldsymbol{E}_{\text {sol }}-\boldsymbol{g}_{n, s o l}\right\|^{2} \\
& \geq\left\|\boldsymbol{E}_{\text {sol }}-\boldsymbol{g}_{n, s o l}\right\|^{2},
\end{aligned}
$$

where $\boldsymbol{g}_{n, i r r}$ and $\boldsymbol{g}_{n, s o l}$ are the irrotational and the solenoidal parts of $\boldsymbol{g}_{n}$, respectively. Expressing Eq. (15) as $\boldsymbol{f}_{n+1}=$ $\boldsymbol{E}_{\text {sol }}+\boldsymbol{g}_{n, i r r}$, the right-hand side of Eq. (47) is further rewritten as

$$
\begin{aligned}
\left\|\boldsymbol{E}_{\text {sol }}-\boldsymbol{g}_{n, \text { sol }}\right\|^{2} & =\left\|\left(\boldsymbol{E}_{\text {sol }}+\boldsymbol{g}_{n, i r r}\right)-\left(\boldsymbol{g}_{n, s o l}+\boldsymbol{g}_{n, i r r}\right)\right\|^{2} \\
& =\left\|\boldsymbol{f}_{n+1}-\boldsymbol{g}_{n}\right\|^{2} .
\end{aligned}
$$

From Eqs. (47) and (48), $\boldsymbol{f}_{n+1}$ is the nearest neighbor of $\boldsymbol{g}$ in $C_{1}$, and hence the projection defined in Eq. (15) is the orthogonal projection onto $C_{1}$.

\section{F. Expression of $\nabla \times \mathrm{H}$ with $\mathrm{H}^{+}, \mathrm{H}^{-}$and $\mathrm{H}_{z}$}

The $x$ - and $y$-components of $\nabla \times \boldsymbol{H}$ can be written as

$$
\begin{aligned}
{[\nabla \times \boldsymbol{H}]_{x} } & =\partial_{y} H_{z}-\partial_{z} H_{y} \\
& =\partial_{y} H_{z}-\partial_{z}\left(-i\left(H^{+}-H^{-}\right)\right) \\
& =\partial_{y} H_{z}+i \partial_{z}\left(H^{+}-H^{-}\right), \\
{[\nabla \times \boldsymbol{H}]_{y} } & =\partial_{z} H_{x}-\partial_{x} H_{z} \\
& =\partial_{z}\left(H^{+}+H^{-}\right)-\partial_{x} H_{z} .
\end{aligned}
$$

The $z$-component of $\nabla \times \boldsymbol{H}$ can be written as

$$
\begin{aligned}
{[\nabla \times \boldsymbol{H}]_{z} } & =\partial_{x} H_{y}-\partial_{y} H_{x} \\
& =-i \partial_{x}\left(H^{+}-H^{-}\right)-\partial_{y}\left(H^{+}+H^{-}\right) \\
& =-i\left(\partial_{x}-i \partial_{y}\right) H^{+}+i\left(\partial_{x}+i \partial_{y}\right) H^{-} \\
& =-2 i \partial H^{+}+2 i \bar{\partial} H^{-} .
\end{aligned}
$$

Also, Gauss's law $(\nabla \cdot \boldsymbol{H}=0)$ can be expressed with $H^{+}, H^{-}$ and $H_{z}$ as

$$
\begin{aligned}
\nabla \cdot \boldsymbol{H} & =\partial_{x} H_{x}+\partial_{y} H_{y}+\partial_{z} H_{z} \\
& =\partial_{x}\left(H^{+}+H^{-}\right)+\partial_{y}\left(-i\left(H^{+}-H^{-}\right)\right)+\partial_{z} H_{z} \\
& =\left(\partial_{x}-i \partial_{y}\right) H^{+}+\left(\partial_{x}+i \partial_{y}\right) H^{-}+\partial_{z} H_{z} \\
& =2 \partial H^{+}+2 \bar{\partial} H^{-}+\partial_{z} H_{z}=0 .
\end{aligned}
$$

Then, substituting Gauss's law into Eq. (51), the $z$-component of $\nabla \times \boldsymbol{H}$ can be also expressed as

$$
[\nabla \times \boldsymbol{H}]_{z}=-4 i \partial H^{+}-i \partial_{z} H_{z} .
$$

Therefore, each component of $\nabla \times \boldsymbol{H}$ can be expressed as

$$
\begin{aligned}
\nabla \times \boldsymbol{H}= & {\left[\begin{array}{c}
\partial_{y} H_{z}+i \partial_{z}\left(H^{+}-H^{-}\right) \\
\partial_{z}\left(H^{+}+H^{-}\right)-\partial_{x} H_{z} \\
-2 i \partial H^{+}+2 i \bar{\partial} H^{-}
\end{array}\right] } \\
= & {\left[\begin{array}{c}
\partial_{y} H_{z}+i \partial_{z}\left(H^{+}-H^{-}\right) \\
\partial_{z}\left(H^{+}+H^{-}\right)-\partial_{x} H_{z} \\
-4 i \partial H^{+}-i \partial_{z} H_{z}
\end{array}\right] . }
\end{aligned}
$$

\section{ACKNOWLEDGEMENTS}

This work was supported by JSPS KAKENHI Grant Number 19H04438 and by Canon Medical Systems Corporation.

\section{REFERENCES}

[1] J. L. Mueller, D. Isaacson, and J. C. Newell: "A reconstruction algorithm for electrical impedance tomography data collected on rectangular electrode arrays," IEEE Transactions on Biomedical Engineering, vol. 46, no. 11 , pp. 1379-1386, 1999.

[2] C. M. Collins, W. Liu, J. Wang, R. Gruetter, J. T. Vaughan, K. Ugurbil, and M. B. Smith: "Temperature and sar calculations for a human head within volume and surface coils at 64 and $300 \mathrm{mhz}$," Journal of Magnetic Resonance Imaging: An Official Journal of the International Society for Magnetic Resonance in Medicine, vol. 19, no. 5, pp. 650-656, 2004.

[3] H. Griffiths: "Magnetic induction tomography," Measurement science and technology, vol. 12, no. 8, p. 1126, 2001.

[4] O. Kwon, E. J. Woo, J.-R. Yoon, and J. K. Seo: "Magnetic resonance electrical impedance tomography (mreit): simulation study of $\mathrm{j}$ substitution algorithm," IEEE Transactions on Biomedical Engineering, vol. 49, no. 2, pp. 160-167, 2002.

[5] U. Katscher, and C. A. van den Berg: "Electric properties tomography: biochemical, physical and technical background, evaluation and clinical applications," NMR in Biomedicine, vol. 30, no. 8, p. e3729, 2017.

[6] E. Haacke, L. Petropoulos, E. Nilges, and D. Wu: "Extraction of conductivity and permittivity using magnetic resonance imaging," Physics in Medicine \& Biology, vol. 36, no. 6, p. 723, 1991.

[7] H. Wen: "Noninvasive quantitative mapping of conductivity and dielectric distributions using if wave propagation effects in high-field mri," in Medical Imaging 2003: Physics of Medical Imaging, vol. 5030. International Society for Optics and Photonics, 2003, pp. 471-477.

[8] E. Insko, and L. Bolinger: "Mapping of the radiofrequency field," Journal of magnetic resonance. Series A (Print), vol. 103, no. 1, pp. 82-85, 1993.

[9] S. Gavazzi, Y. Shcherbakova, L. W. Bartels, L. J. Stalpers, J. J. Lagendijk, H. Crezee, C. A. van den Berg, and A. L. van Lier: "Transceive phase mapping using the planet method and its application for conductivity mapping in the brain," Magnetic resonance in medicine, vol. 83, no. 2, pp. 590-607, 2020.

[10] U. Katscher, T. Voigt, C. Findeklee, P. Vernickel, K. Nehrke, and O. Doessel: "Determination of electric conductivity and local sar via b1 mapping," IEEE transactions on medical imaging, vol. 28, no. 9, pp. 1365-1374, 2009.

[11] J. K. Seo, M.-O. Kim, J. Lee, N. Choi, E. J. Woo, H. J. Kim, O. I. Kwon, and D.-H. Kim: "Error analysis of nonconstant admittivity for mrbased electric property imaging," IEEE transactions on medical imaging, vol. 31, no. 2, pp. 430-437, 2011.

[12] F. S. Hafalir, O. F. Oran, N. Gurler, and Y. Z. Ider: "Convection-reaction equation based magnetic resonance electrical properties tomography (crmrept)," IEEE transactions on medical imaging, vol. 33, no. 3, pp. 777793, 2014.

[13] T. Nara, T. Furuichi, and M. Fushimi: "An explicit reconstruction method for magnetic resonance electrical property tomography based on the generalized cauchy formula," Inverse Problems, vol. 33, no. 10, 105005, 2017.

[14] E. Balidemaj, C. A. van den Berg, J. Trinks, A. L. van Lier, A. J. Nederveen, L. J. Stalpers, H. Crezee, and R. F. Remis: "Csi-ept: a contrast source inversion approach for improved mri-based electric properties tomography," IEEE transactions on medical imaging, vol. 34, no. 9, pp. 1788-1796, 2015.

[15] R. L. Leijsen, W. M. Brink, C. A. Van Den Berg, A. G. Webb, and R. F. Remis: "3-d contrast source inversion-electrical properties tomography," IEEE transactions on medical imaging, vol. 37, no. 9, pp. 2080-2089, 2018.

[16] R. Hong, S. Li, J. Zhang, Y. Zhang, N. Liu, Z. Yu, and Q. H. Liu: "3-d mri-based electrical properties tomography using the volume integral equation method," IEEE Transactions on Microwave Theory and Techniques, vol. 65, no. 12, pp. 4802-4811, 2017.

[17] L. Guo, J. Jin, M. Li, Y. Wang, C. Liu, F. Liu, and S. Crozier: "Reference-based integral mr-ept: Simulation and experiment studies at 9.4 t mri," IEEE Transactions on Biomedical Engineering, vol. 66, no. 7, pp. 1832-1843, 2018.

[18] J. Liu, X. Zhang, S. Schmitter, P.-F. Van de Moortele, and B. He: "Gradient-based electrical properties tomography (g ept): A robust method for mapping electrical properties of biological tissues in vivo using magnetic resonance imaging," Magnetic resonance in medicine, vol. 74, no. 3, pp. 634-646, 2015.

[19] Y. Wang, Q. Shao, P.-F. Van de Moortele, E. Racila, J. Liu, J. Bischof, and B. He: "Mapping electrical properties heterogeneity of tumor using boundary informed electrical properties tomography (biept) at 7t," Magnetic resonance in medicine, vol. 81, no. 1, pp. 393-409, 2019. 
[20] N. Hampe, M. Herrmann, T. Amthor, C. Findeklee, M. Doneva, and U. Katscher: "Dictionary-based electric properties tomography," Magnetic resonance in medicine, vol. 81, no. 1, pp. 342-349, 2019.

[21] S. Mandija, E. F. Meliadò, N. R. Huttinga, P. R. Luijten, and C. A. van den Berg: "Opening a new window on mr-based electrical properties tomography with deep learning," Scientific reports, vol. 9, no. 1, pp. 1-9, 2019.

[22] A. Nachman, D. Wang, W. Ma, and M. Joy: "A local formula for inhomogeneous complex conductivity as a function of the rf magnetic field," in Proceedings of the 15th Scientific Meeting of the International Society of Magnetic Resonance in Medicine (ISMRM' 07), vol. 15. Citeseer, 2007.

[23] W. Freeden, and C. Gerhards:, Geomathematically oriented potential theory. CRC press, 2012.

[24] Y. F. Gui, and W.-B. Dou: "A rigorous and completed statement on helmholtz theorem," Progress in Electromagnetics Research, vol. 69, pp. 287-304, 2007.

[25] A. Kustepeli: "On the helmholtz theorem and its generalization for multilayers," Electromagnetics, vol. 36, no. 3, pp. 135-148, 2016.

[26] D. C. Youla, and H. Webb: "Image Restoration by the Method of Convex Projections: Part1 Theory," IEEE Transactions on Medical Imaging, vol. 1, no. 2, pp. 81-94, 1982.

[27] F. Vico, L. Greengard, and M. Ferrando: "Fast convolution with freespace green's functions," Journal of Computational Physics, vol. 323, pp. 191-203, 2016.

[28] B. Aubert-Broche, M. Griffin, G. B. Pike, A. C. Evans, and D. L. Collins: "Twenty new digital brain phantoms for creation of validation image data bases," IEEE transactions on medical imaging, vol. 25, no. 11, pp. 1410-1416, 2006.

[29] S. Rajagopalan, and R. A. Robb: "Image smoothing with savitzkygolay filters," in Medical Imaging 2003: Visualization, Image-Guided Procedures, and Display, vol. 5029, 2003, pp. 773-781.

[30] T. Nara, and M. Fushimi: "Computation of $\mathrm{H}-$ from $\mathrm{H}+$ and its application to regularization for magnetic resonance electrical properties tomography (MREPT)," in Proceedings of ISMRM2020, vol. 28, 2020, p. 3186 . 